\title{
Patientenorientierte Notfallsteuerung
}

Robert Messerle, Jonas Schreyögg und Ferdinand M. Gerlach

\author{
Inhaltsverzeichnis
}

\subsection{Einleitung - 44}

3.2 Herausforderungen in der Notfallversorgung - 46

3.2.1 Informations- und Steuerungsdefizite - 47

3.2.2 Strukturelle Probleme - 48

3.2.3 Internationale Einordnung - 49

3.3 Lösungsansätze für eine patientenorientierte Notfallsteuerung - $\mathbf{5 0}$

3.3.1 Integrierte Leitstellen - 52

3.3.2 Integrierte Notfallzentren - 54

3.3.3 Rettungsdienst - 57

3.3.4 Patientenpfade -59

3.4 Politische Entwicklungen - 60

3.5 Fazit -62

Literatur - 62 


\section{- Zusammenfassung}

Der Referentenentwurf zur Notfallversorgung wurde kurz vor einer der größten Krisen der Bundesrepublik veröffentlicht. Strukturelle Probleme des deutschen Gesundheitswesens wurden in der Krise besonders sichtbar und mussten quasi über Nacht zumindest provisorisch gelöst werden. Schon zuvor legten steigende Fallzahlen und eine weitgehend fehlende Steuerung die Probleme der Notfallversorgung offen. Eine grundlegende Strukturreform zur längerfristigen Behebung der verschiedenen Defizite erscheint einmal mehr notwendig, wurde aber vom Gesetzgeber zunächst vertagt.

Der Sachverständigenrat stellte dazu bereits 2018 umfassende Empfehlungen vor, welche hier vorgestellt und punktuell ergänzt werden. Das Leitbild sind sektorenübergreifend koordinierte, klar abgestufte Versorgungspfade. In Integrierten Leitstellen (ILS) beurteilen erfahrene Fachkräfte rund um die Uhr die Behandlungsdringlichkeit der Anrufer und lenken die Versorgung in bedarfsgerechte und effiziente Strukturen. Ebenfalls rund um die Uhr erreichbare Integrierte Notfallzentren (INZ) stellen an qualitativ besonders geeigneten Kliniken den ersten Anlaufpunkt dar. Die weitere Behandlung erfolgt aus einer Hand, ambulant oder stationär.

The draft bill on emergency care was published shortly before one of the biggest crises in the history of Germany. Structural problems of the German health care system became particularly evident during the corona crisis and had to be resolved - at least provisionally - almost overnight. Even before that, rising numbers of cases and a lack of patient guidance revealed the shortcomings of emergency care. A fundamental structural reform to remedy the various deficits appears very necessary, but was postponed by the legislature.

The Advisory Council on the Assessment of Developments in the Health Care System (SVR) presented comprehensive recommendations on this issue as early as 2018. These suggestions are presented in this paper and supplemented at certain points. The guiding principle are cross-sectoral coordinated tiered clinical pathways. Experienced specialists in integrated control centers (ILS) carry out a qualified initial assessment (i.e. triage) around the clock and direct the patients into needs-based and efficient care structures. Integrated emergency centres (INZ), located at high-quality clinics and also available around the clock, are the first point of contact. The subsequent treatment is provided from a single source, either on an outpatient or inpatient basis.

\subsection{Einleitung}

Als Notfall wird im Allgemeinen eine unerwartete Situation verstanden, in der schnelle Hilfe notwendig ist. Die auf den ersten Blick simpel erscheinende Frage, was einen Notfall in der medizinischen Versorgung charakterisiert, lässt sich jedoch kaum allgemeingültig beantworten und ist stark von der eingenommenen Perspektive abhängig (Wolcott 1979). Dementsprechend reicht die Definition eines Notfalls von der (zumeist juristischen) Beschränkung auf rein lebensbedrohliche Situationen (z. B. BSG Az. B 6 KA 1/12 R) bis zur Berücksichtigung von akuten, lediglich geringfügigen Gesundheitsproblemen, deren unverzügliche Betreuung aber aus subjektiver Patientensicht notwendig ist (z.B. Behringer et al. 2013).

An der Behandlung der Not- und Akutfälle nehmen in Deutschland grundsätzlich drei Versorgungsbereiche (• Abb. 3.1) mit in der Theorie unterschiedlichen Aufgaben teil. Die ambulante Versorgung obliegt bei nicht lebensbedrohlichen Fällen den niedergelassenen Ärztinnen und Ärzten im Rahmen des Sicherstellungsauftrages ( $\$ 75$ SGB V). Für die Versorgung außerhalb der Sprechzeiten organisieren die Kassenärztlichen Vereinigungen (KV) den ärztlichen Bereitschaftsdienst (ÄBD). Der Rettungsdienst ist für die not- 


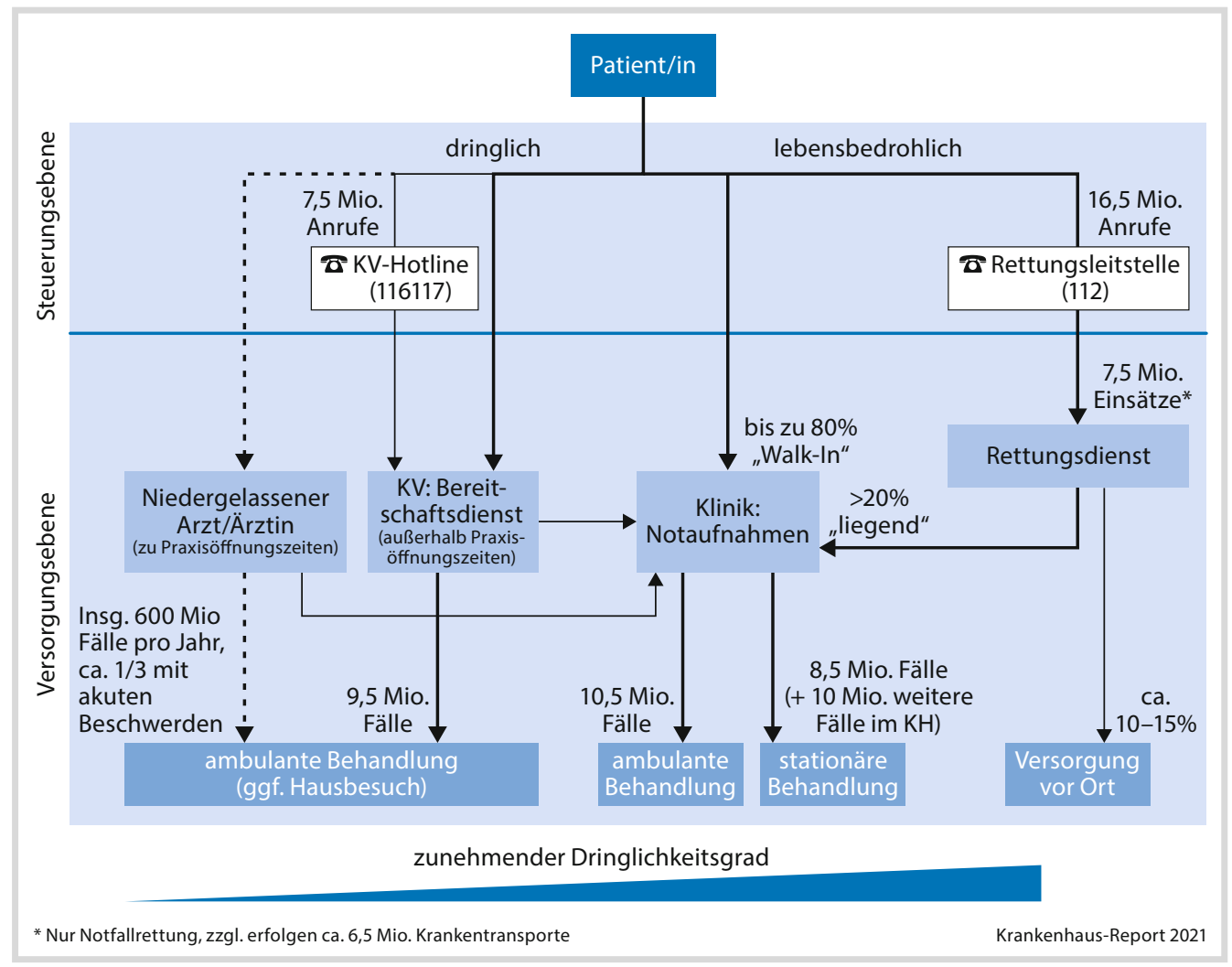

- Abb. 3.1 Derzeitige Struktur der Notfallversorgung in Deutschland (Quelle: Grafisch modifiziert nach SVR 2018. Die Daten zum Fallaufkommen beruhen auf Destatis 2019; Europäische Kommission 2020; Trentzsch et al. 2019; Zi 2019b. Angaben für den Rettungsdienst sind nur vereinzelt, z. B. für einzelne Bundesländer und Kreise, oder als Hochrechnung verfügbar. Herangezogen wurden Günther et al. 2017; Schmiedel und Behrendt 2019; SQR-BW 2020. Alle Angaben sind auf 500.000 gerundet. Die Zahlen der stationären Notfälle und zum Rettungsdienst umfassen auch PKVVersicherte, alle anderen Angaben nur GKV-Versicherte. Mit gestrichelten Pfeilen sind Behandlungsmöglichkeiten gekennzeichnet, die üblicherweise nicht zur Notfallversorgung gezählt werden, in denen aber dennoch die Versorgung zumindest akuter Fälle stattfindet.)

fallmedizinische Versorgung vor Ort zur Abwendung von Lebensgefahr und schweren gesundheitlichen Schäden, die qualifizierte Beförderung der Patientinnen und Patienten ins Krankenhaus (,Notfallrettung“) sowie für den allgemeinen Krankentransport zuständig. Die Notaufnahmen der Krankenhäuser übernehmen die Anschlussversorgung an den Rettungsdienst und dienen als Zugang zur weiteren stationären Versorgung. Sie stellen jedoch auch eine direkte Anlaufstelle dar und behandeln Patientinnen und Patienten ambulant.
Mit der Notrufnummer (112) und der Rufnummer des ÄBD (116117) existieren zwar Ansätze zur Steuerung, jedoch steht es den Patientinnen und Patienten letztendlich frei, die aus ihrer Sicht passende Versorgungsebene auszuwählen (siehe Abschn. 3.2). Die Angaben zur Inanspruchnahme zeigen, dass - außerhalb der Regelversorgung bei den niedergelassenen Ärztinnen und Ärzten - vor allem den klinischen Notaufnahmen eine zentrale Rolle zukommt. Sie behandeln mit ca. 10,5 Mio. Fällen mehr als $50 \%$ der ambulanten Notfälle (Zi 2019b). Sie stellen außerdem den Zugang 


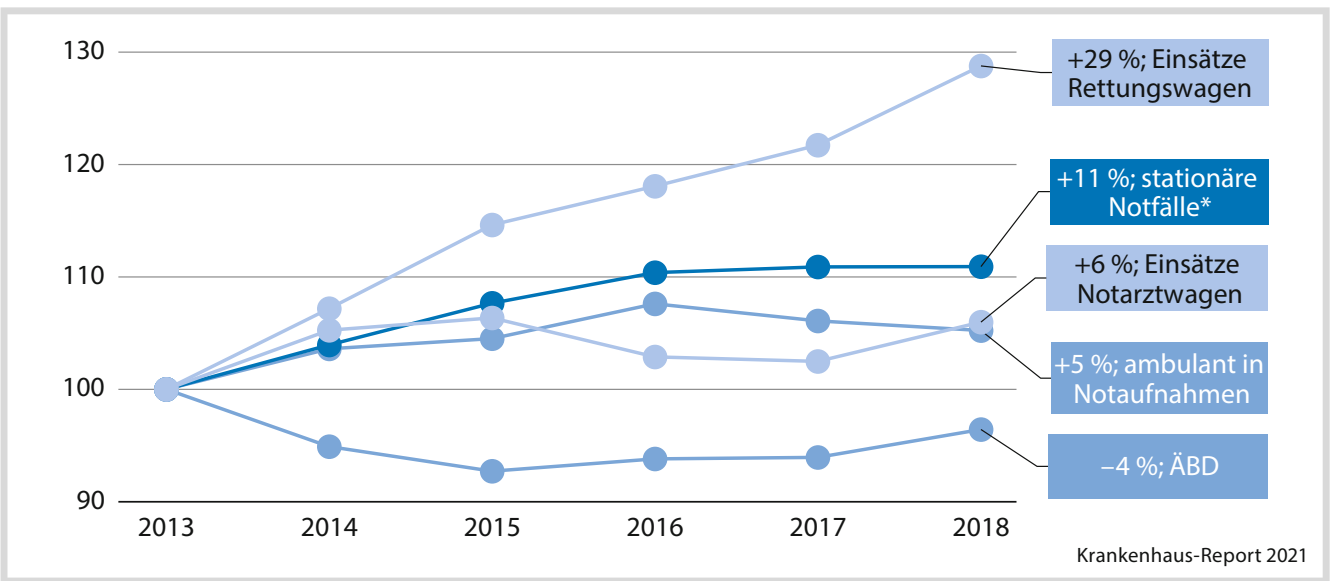

- Abb. 3.2 Veränderung der Inanspruchnahme in der Akut- und Notfallversorgung seit 2013, in \% (Quelle: Eigene Darstellung basierend auf BMG 2020a; Destatis 2019; Zi 2019a, 2019b. *Die Zahlen der stationären Notfälle umfassen auch PKV-Versicherte, alle anderen Angaben nur GKV-Versicherte)

für jährlich etwa 8,5 Mio. stationäre Notfälle dar. ${ }^{1}$

Die behandelten Indikationen und die Art der erbrachten Leistungen unterscheiden sich zwischen dem ÄBD und den Notaufnahmen. Notaufnahmen behandeln ein relativ homogenes Indikationsspektrum - vorrangig Verletzungen - und setzen häufig diagnostische bzw. bildgebende Verfahren ein. Im ÄBD wird hingegen ein deutlich breiteres Behandlungsspektrum abgedeckt ${ }^{2}$, das eher selten mit technischen Leistungen verbunden ist (Dräther und Schäfer 2017).
Die in den letzten Jahren zu beobachtende stetige Verlagerung der Versorgung in die Notaufnahmen scheint vorerst gestoppt (- Abb. 3.2). Die Inanspruchnahme hat sich in den meisten Bereichen stabilisiert. Eine Ausnahme bildet der Rettungsdienst. Dessen Nutzung steigt, insbesondere ohne Beteiligung eines Notarztes, weiterhin stark an. Im Gegenzug nehmen die durchschnittlichen Zeiten bis zum Eintreffen am Einsatzort seit Jahren zu (Schmiedel und Behrendt 2019).

\subsection{Herausforderungen in der Notfallversorgung}

Der kurze Überblick zur Struktur und Inanspruchnahme verrät bereits, dass an verschiedenen Stellen der Notfallversorgung Defizite vorliegen. Auf der einen Seite bestehen strukturelle Probleme, infolge derer die zur bedarfsgerechten Versorgung benötigten Mittel nicht zur Verfügung stehen. Auf der anderen Seite fehlt es an Information und Steuerung, wodurch auch die bestehenden Strukturen nicht bedarfsgerecht genutzt werden. 


\subsubsection{Informations- und Steuerungsdefizite}

Notfälle stellen u. a. aufgrund der nur beschränkten Planbarkeit einen hohen Anspruch an die Steuerung der Versorgung dar. Da keine klare Aufgabentrennung zwischen den Sektoren existiert und Strukturen teilweise mehrfach vorgehalten werden, ist im Status quo jedoch letztendlich die Entscheidung der Patienten für den weiteren Versorgungsablauf maßgeblich. ${ }^{3}$

Doch bei diesen herrscht oftmals Unwissenheit sowohl über die Strukturen der Notfallversorgung und die vorgesehenen Versorgungspfade (Somasundaram et al. 2018) als auch über wichtige Leitsymptome klinischer Notfälle (Luiz et al. 2017). Innerhalb und außerhalb der Praxissprechzeiten ist daher das Krankenhaus der erste Ansprechpartner in der Not (KBV 2020; kkvd 2017). In städtischen Regionen kommen bis zu $80 \%$ der Notaufnahmepatienten selbstständig (,zu Fuß“) zur Klinik (Trentzsch et al. 2019). Sie selbst verstehen sich in der Regel als Not- bzw. zumindest als Akutfall. Diese Selbsteinstufung weicht jedoch oft von der professionellen Einschätzung ab. Aus medizinischer Sicht besteht im Schnitt bei etwa der Hälfte dieser Patientinnen und Patienten nur eine geringe Dringlichkeit (Reinhold et al. 2020; Trentzsch et al. 2019).

Der Einsatz der Leitstellen als Steuerungsinstrument gestaltet sich herausfordernd. Trotz verstärkter Informationskampagnen ist die 116117, die bis vor kurzem nur zu bestimmten Uhrzeiten erreichbar war, lediglich $20 \%$ der Bevölkerung bekannt (KBV 2020). Die 112 ist zwar deutlich geläufiger (etwa $80 \%$, PwC 2019), sie ist jedoch auf schwerwiegende und zeitkritische Not- und Unfälle fokussiert. Für die differenzierte Steuerung weniger dringlicher Akutfälle sind die Ret-

3 Eine Beteiligung der Patientinnen und Patienten ist im Sinne einer partizipativen Entscheidungsfindung sinnvoll (SVR 2018). Jedoch sollte diese auf informierter Basis erfolgen - was im Fall der Notfallversorgung durchaus bezweifelt werden kann. tungsleitstellen in der Regel weder spezifiziert noch ausgestattet. So sind z. B. strukturierte Notrufabfragen nicht flächendeckend implementiert (Luiz et al. 2019). Bei bis zu $60 \%$ der vom Rettungsdienst aufgesuchten Patientinnen und Patienten liegt retrospektiv keine akut lebensbedrohliche Situation vor (Lechleuthner et al. 2019). Hier könnte die bisher nicht verbindliche Zusammenarbeit beider Rufnummern inkl. einer koordinierten Anrufübergabe helfen.

Eine Behandlung in der vertragsärztlichen Versorgung käme für viele Patientinnen und Patienten dabei auch aus subjektiver Sicht in Frage. Wahrgenommene Zugangsbarrieren (,Terminschwierigkeiten“) in der ambulanten Versorgung und die Erwartung besserer Behandlungsmöglichkeiten führen jedoch insbesondere bei jüngeren Patienten zur Vorstellung in der Notaufnahme, obwohl andere Behandlungsoptionen ggf. besser geeignet wären (Scherer et al. 2017; Somasundaram et al. 2018). Dies geht zum Teil so weit, dass sie eher eine Notaufnahme in der nächsten Stadt als niedergelassene Ärzte bzw. den ÄBD in der Nähe aufsuchen (Reinhold et al. 2020).

Doch auch niedergelassene Ärzte schicken ihre Patienten unter der Annahme besserer Ressourcen zur weiteren Diagnostik und Behandlung in die Notaufnahmen (Oslislo et al. 2019; Schmiedhofer et al. 2017). Denn die Einschätzung der Behandlungsdringlichkeit, oft anhand unspezifischer Leitsymptome, stellt auch Fachkräfte vor eine Herausforderung. Diese Unwägbarkeit führt zusammen mit Angst vor Fehlentscheidungen und deren rechtlichen Folgen dazu, dass auch ,Bagatellpatienten" diagnostischen und therapeutischen Aufwand verursachen und zur starken Auslastung der Strukturen beitragen (Searle et al. 2015).

Der Rettungsdienst sieht sich ähnlichen Herausforderungen gegenüber. Der Anteil der Notarzteinsätze nimmt stetig ab; nur an etwa $40 \%$ der Notfälle ist noch ein Notarzt beteiligt. Über $10 \%$ der Notfalleinsätze werden ohne Sonderrechte (vereinfacht gesagt ,ohne Blaulicht und Sirene") gefahren (Schmiedel 
und Behrendt 2019). Dort, wo Leerfahrten finanziert werden (siehe unten), wird ein stetig zunehmender Anteil der Patienten gar nicht erst transportiert, sondern ambulant behandelt (SQR-BW 2020). Auch scheinen teilweise eher pflegerische und weniger notfallmedizinische Aspekte Grund von Einsätzen zu sein (SVR 2018). Bei im Rettungsdienst tätigen Fachkräften besteht daher weitestgehend Einigkeit, dass hinter der wachsenden Inanspruchnahme im Wesentlichen keine unmittelbar lebensbedrohlichen Erkrankungen stehen. Aufgrund der in den meisten Bundesländern dürftigen Datenlage im Rettungsdienst sind genauere Aussagen aber nur schwer möglich (Lechleuthner 2017).

\subsubsection{Strukturelle Probleme}

Die dargelegten Steuerungs- und Informationsdefizite werden durch strukturelle Probleme verschärft. Für eine bedarfsgerechte Steuerung sind kaum Versorgungspfade verfügbar.

Das Fehlen abgestufter Behandlungsmöglichkeiten beginnt bereits in den Leitstellen. Diesen steht nur eine sehr begrenzte Auswahl zur Disposition. Die Entsendung eines Krankentransports unterliegt der ärztlichen Verordnung, der ÄBD und niedergelassene Ärzte sind nicht eingebunden. Es verbleibt oft nur die Frage, ob zusätzlich zum Rettungswagen ein Notarzt alarmiert wird. An der Schnittstelle zur klinischen Versorgung ergeben sich weitere Probleme. Notfallmedizinische Leitlinien sehen den Transport in das ,nächste geeignete Krankenhaus" vor. Die Behandlung mit spezialisierten Ressourcen verspricht bessere Behandlungsergebnisse als in einem für die konkrete Versorgung nicht geeigneten, aber dafür nahen Krankenhaus (z. B. Nimptsch und Mansky 2017). Abhängig von der Indikation können z. B. der Zugriff auf eine Stroke Unit oder die Kapazitäten eines Traumazentrums entscheidend sein (Fischer et al. 2016).

Etwa ein Drittel der Krankenhäuser hat aber nicht einmal Kapazitäten zur intensiv- medizinischen Versorgung; viele, vor allem kleinere Krankenhäuser haben kein CT (Destatis 2017). Selbst vorhandene Ressourcen können oft nicht vollständig genutzt werden. Eine Umfrage legt nahe, dass Notfall- und Operationskapazitäten in vier von fünf Krankenhäusern zumindest gelegentlich aus Personalmangel eingeschränkt sind (Karagiannidis et al. 2019). Die Wahl eines Krankenhauses mit geeigneten und verfügbaren Kapazitäten ist daher elementar wichtig. Welches dazu zählt ist jedoch mangels strukturierter und zentraler Dokumentation der sehr heterogenen Versorgungsstrukturen und deren Auslastung häufig weder für den Rettungsdienst noch für die Leitstellen klar. E-Health-Anwendungen wie IVENA setzen an dieser Schnittstelle an und ermöglichen den Zugriff auf Kapazitäten und Auslastungen der Krankenhäuser sowie den Austausch elektronischer Patientendaten (Walter und Fischer 2017), werden jedoch nicht deutschlandweit eingesetzt. Die Abmeldung von Kapazitäten erfolgt daher z. T. noch telefonisch oder per Fax (Niedersächsischer Landtag 2015). Dieser unzureichende Informationsaustausch kennzeichnet nicht nur die Schnittstelle von Präklinik zur Klinik, sondern ist symptomatisch für die gesamte Notfallversorgung.

Für nicht lebensbedrohlichen Behandlungsbedarf stehen die niedergelassenen Ärzte und der ÄBD - auch durch Hausbesuche zur Verfügung. Sie sind jedoch in die Steuerung der Notfallversorgung kaum eingebunden und die Organisation des ÄBD sowie der Kooperationsgrad mit den Kliniken differiert trotz verschiedener Reformen weiterhin stark (Schmidt und Wildner 2019). Die Portal- bzw. Notdienstpraxen sind je nach Wochentag und Region unterschiedlich erreichbar und an normalen Praxistagen z. T. nur für zwei Stunden geöffnet. Dies ist auch darauf zurückzuführen, dass sich der durchgängige Betrieb einer Portalpraxis ohne weitere Anpassung der Versorgungsstrukturen nur an wenigen Standorten wirtschaftlich lohnt (Augurzky et al. 2018).

Die Leitstellen und der Rettungsdienst stehen zusätzlich vor dem Spagat, einerseits in das System der Gefahrenabwehr bzw. die Ver- 
sorgung bei Großschadensereignissen eingebunden zu sein, andererseits jedoch im Regelbetrieb auch wenig dringliche Akutfälle versorgen zu müssen. Folge dieser auseinanderlaufenden Anforderungen ist - wie auch im ÄBD - eine sehr heterogene Besetzung und Qualifikation des Leitstellenpersonals (Hackstein et al. 2015; Breckner et al. 2020). Im Rettungsdienst wurde hingegen mit dem Notfallsanitäter ein deutschlandweit einheitliches Berufsbild geschaffen. Die Ausübung des Berufs unterliegt bei heilkundlichen Maßnahmen jedoch großer rechtlicher Unsicherheit. Um dieses Dilemma zu umgehen, werden auf der Ebene des Rettungsdienstträgers oder Bundeslandes ergänzende Regelungen getroffen (vgl. z. B. Breuer et al. 2020). In der Folge unterscheiden sich die Kompetenzen der Notfallsanitäter sowohl zwischen den Bundesländern (Deutscher Bundestag 2020) als auch zwischen den Rettungsdienstbereichen (Mann et al. 2020)

Die beschriebenen Defizite sind auch dadurch bedingt, dass eine gemeinsame, übergreifende Planung fehlt. Jeder Bereich plant einzeln: Die Organisation des Rettungsdienstes folgt der Aufgabenträgerschaft und wird zumeist auf Kreisebene für etwa 300 Rettungsdienstbereiche getrennt durchgeführt (Augurzky et al. 2015). Den ÄBD organisieren die KVen in eigener Verantwortung. Die Krankenhausplanung übernehmen die Bundesländer. Für die Notfallversorgung erfolgt dabei kaum bzw. keine Zielsetzung. So wird bisher auf die Vorgaben des Gemeinsamen Bundesausschusses (G-BA) zu gestuften Notfallstrukturen nur in drei Bundesländern Bezug genommen (Stand Dezember 2019, DKG 2019).

Eine Reihe finanzieller Fehlanreize verschärft die Situation. Zunächst ist der Erstattungsanspruch für Rettungsfahrten gegenüber den Krankenkassen in der Regel an die Verknüpfung mit einer medizinischen Leistung - wie die Weiterversorgung im Krankenhaus - gebunden (BSG Az. 1 KR 38/07 R). ${ }^{4}$

4 Inwieweit dies infolge regionaler Vereinbarungen noch gilt, ist umstritten (vgl. BAND 2020).
Für Patienten und Rettungsdienstträger ist der Transport ins Krankenhaus anstelle einer ,Leerfahrt“ aus finanzieller Sicht daher oft die bessere Wahl. In der Notaufnahme angekommen, ist die ambulante Vergütung aus Sicht der Krankenhäuser nicht kostendeckend (Haas et al. 2015), sodass die Notaufnahmen zunehmend die Eintrittspforte für stationäre Aufnahmen darstellen. Erschwert wird die Situation dadurch, dass die ambulante Notfallversorgung - dem Sicherstellungsauftrag folgend - direkt aus dem ,Budget“ (der morbiditätsbedingten Gesamtvergütung) der niedergelassenen Ärzte finanziert wird ( $\$ 87 b$ Abs. 1 SGB V). Jeder ambulant in einer Notaufnahme behandelte Fall senkt also die für die vertragsärztliche Vergütung zur Verfügung stehenden Mittel. Entsprechend scharf sind die Diskussionen zwischen den Beteiligten und entsprechend groß die Vorbehalte hinsichtlich einer stärkeren Kooperation.

Im Ergebnis werden in Notaufnahmen und im Rettungsdienst auch Patientinnen und $\mathrm{Pa}-$ tienten versorgt, die diese spezialisierten Ressourcen nicht benötigen und deren Behandlung durch abgestufte Versorgungsangebote bedarfsgerechter und gleichzeitig effizienter erfolgen könnte. Als Resultat der undifferenzierten Inanspruchnahme der Versorgungstrukturen stehen neben unzufriedenem und überlastetem Personal erhöhte Warte- und Behandlungszeiten sowie schlechtere Behandlungsergebnisse bis hin zu einer erhöhten Mortalität (Morley et al. 2018).

\subsubsection{Internationale Einordnung}

Die Notfallversorgung steht nicht nur in Deutschland vor großen Herausforderungen, ähnliche Probleme werden auch international seit vielen Jahren diskutiert (Graff 1999; Miles et al. 2017). Dementsprechend stoßen auch andere Länder Reformprozesse an (siehe z. B. Baier et al. 2019; Roßbach-Wilk et al. 2019 für Übersichten). 
Inhalt solcher Bestrebungen ist oft, den $\mathrm{Zu}$ gang zum primärärztlichen Versorgungsangebot für Akutfälle zu erleichtern. Dies wird z. B. durch rund um die Uhr verfügbare Hotlines mit telefonischer ärztlicher Beratung und digitale Informationsplattformen versucht. AuBerdem werden die verfügbaren Ressourcen der Akut- und Notfallversorgung in größeren Versorgungseinheiten an oder in der Nähe von Kliniken konzentriert. Die Versorgung von Notfällen erfolgt dort in der Regel durch speziell weitergebildete Notfallmediziner, für besonders schwere Behandlungsbilder übernehmen Zentren mit spezialisierten Kapazitäten die Versorgung. Vorreiter in diesem Konzentrationsprozess ist vermutlich Dänemark, das die Notfallversorgung in landesweit nur 21 Notaufnahmen zentriert. ${ }^{5}$ Für die adäquatere Behandlung von weniger dringlichen Fällen werden international die Akut- und Notfallversorgung sowohl räumlich als auch organisatorisch stärker verschränkt und die Patientinnen und Patienten durch eine frühzeitige Auskunft und Beratung in bedarfsgerechte Strukturen gelenkt.

Evaluationen dieser Reformen ergeben auch infolge der komplexen Ursachen und der im Regelfall nicht singulären Maßnahmen ein eher gemischtes Bild (Cooper et al. 2019; Foster et al. 2020; Gonçalves-Bradley et al. 2018; van den Heede und van de Voorde 2016). Die Betrachtung einzelner Vorreiter wie Dänemark ermöglicht ebenfalls keine klare Schlussfolgerung. Dort werden infolge der regionalen Ausgestaltung sehr unterschiedliche Entwicklungen gemeldet ${ }^{6}$. Im Ergebnis unterstreichen

5 Allerdings werden die 21 Notaufnahmen noch nicht durchgängig mit eigenen angestellten Fachkräften (z. B. für Notfallmedizin) besetzt. Stattdessen wird insbesondere nachts und am Wochenende auf ein ,virtuelles Modell“ zurückgegriffen, in dem Assistenzärzte aus anderen Fachabteilungen des Krankenhauses die Notfallversorgung sicherstellen (Moellekaer et al. 2019).

6 Als Folge der systematischen Patientensteuerung wurden aus mehreren dänischen Regionen sinkende Behandlungszahlen in den Notaufnahmen gemeldet (Ministeriet for Sundhed og Forebyggelse 2014). Neuere Daten zeigen nun eine auseinandergehende Entwick- internationale Erfahrungen, dass ein breites Bündel an Maßnahmen notwendig ist, um eine bedarfsgerechte Notfallfallversorgung zu erreichen.

\subsection{Lösungsansätze für eine patientenorientierte Notfallsteuerung}

Angesichts der breit und intensiv diskutierten Defizite der Notfallversorgung in Deutschland wird ein grundlegender Reformbedarf von allen Beteiligten anerkannt. Verschiedene Positionspapiere mit unterschiedlichen Herangehensweisen wurden vor diesem Hintergrund erarbeitet und im Krankenhaus-Report bereits dargelegt (Slowik et al. 2018). Die im Folgenden präsentierten Lösungsansätze basieren auf dem im Herbst 2017 erstmals vorgestellten und im Sommer 2018 veröffentlichten Konzept des Sachverständigenrates (SVR 2018).

Leitbild des Konzepts ist, dass den Patientinnen und Patienten zukünftig sektorenübergreifend koordinierte, klar abgestufte Versorgungspfade zur Verfügung stehen. Hilfesuchenden soll durch die Versorgung ,aus einer

lung zwischen der Hauptstadtregion und dem Rest Dänemarks (Fløjstrup et al. 2020). Denn in der Hauptstadtregion wurde 2014 gemeinsam mit der Einführung der systematischen Patientensteuerung die Kooperation mit der Vertretung der Allgemeinmediziner aufgehoben. Der Bereitschaftsdienst wurde in der Folge eingestellt und die Anzahl der Hausbesuche deutlich reduziert. Stattdessen wurden die Notaufnahmen als zentrale und alleinige Anlaufstelle etabliert. Die Leitstelle vereinbart seitdem für Patientinnen und $\mathrm{Pa}$ tienten, die einen Arztkontakt benötigen, direkt einen Termin in den neu strukturierten Notaufnahmen (VIVE 2018; Søvsø et al. 2020). Der Umstrukturierung folgte ein sprunghafter Anstieg der Notaufnahmebesuche um $30 \%$, während diese in den anderen Regionen stabil blieben (Fløjstrup et al. 2020). Inwiefern sich die Gesamtbelastung der Notfallversorgung verändert hat und die Mehrbelastung der Notaufnahmen dadurch gerechtfertigt werden kann, ist nicht bekannt. In der Zwischenzeit scheint in der Hauptstadtregion jedoch wieder auf eine stärkere Kooperation mit den Allgemeinmedizinern gesetzt zu werden (vgl. The Capital Region of Denmark 2019). 


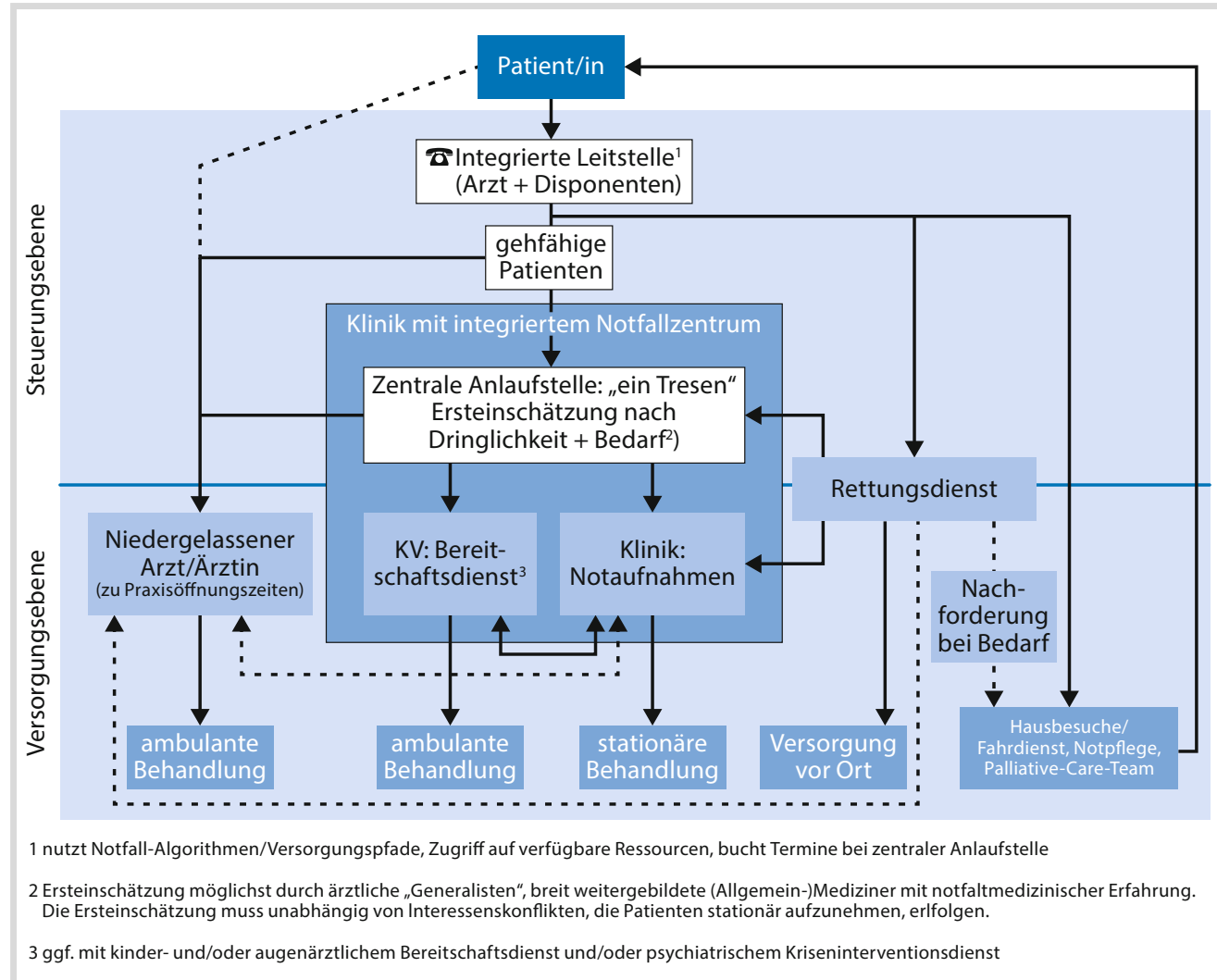

- Abb. 3.3 Konzept des SVR zur Notfallversorgung (Quelle: Grafisch modifiziert nach SVR 2018. Durchgehend gezeichnete Pfeile stellen die regelhaften Versorgungspfade dar. Mit gestrichelten Pfeilen sind Behandlungsmöglichkeiten gekennzeichnet, die genutzt werden können, jedoch seltener auftreten.)

Hand" und die zentrale Beratung und Anleitung eine bedarfsgerechte und dabei effiziente Behandlung gewährleistet werden. Unter Rückgriff auf leitliniengestützte Notfallalgorithmen soll eine objektive Einschätzung der Dringlichkeit, die individuelle Auswahl des besten Versorgungspfades und eine (digitale) Begleitung des weiteren Behandlungsablaufs erfolgen.

Um dies zu erreichen, werden die ambulanten und stationären Strukturen enger verzahnt sowie Versorgungsangebote eingebunden. Der Rettungsdienst wird über die reine Transportleistung hinaus als präklinische Notfallmedizin in das Versorgungssystem integriert. Begleitet werden muss die Umstrukturierung der
Notfallversorgung mit Aufklärungskampagnen und umfangreichen, adressatengerechten $\mathrm{Pa}$ tienteninformationen, damit Transparenz und Verständnis für die neuen Strukturen geschaffen werden. Voraussetzung für eine erfolgreiche Umsetzung der Reformpläne ist die Implementierung einer flächendeckenden digitalen Infrastruktur und eines reibungslosen Informationsaustauschs aller Beteiligten.

- Abb. 3.3 stellt das Konzept überblicksartig dar. Kernstück der neuen Notfallversorgung sind Integrierte Leitstellen (ILS) und Integrierte Notfallzentren (INZ). Die neuen Versorgungspfade basieren auf diesen beiden neuen Elementen und binden darauf aufbauend abgestufte Behandlungsmöglichkeiten ein. 


\subsubsection{Integrierte Leitstellen}

Die Integrierten Leitstellen ${ }^{7}$ übernehmen rund um die Uhr die initiale Koordination der Notfallversorgung. Sowohl Akut- als auch Notfälle werden in den Leitstellen mittels einer standardisierten Notrufabfrage und unter Nutzung digitaler Entscheidungsalgorithmen telefonisch ersteingeschätzt.

Idealerweise werden die Anrufe unter einer deutschlandweit einheitlichen Telefonnummer entgegengenommen, um den Patientinnen und Patienten die Entscheidung abzunehmen, welche die angemessene Versorgungsebene ist. In der Annahme, dass eine gewisse Vorfilterung sinnvoll sein könnte und eine umfangreiche Aufklärung zukünftig zu einer gezielten Nutzung der 112 oder der 116117 führt, erscheint jedoch auch die Beibehaltung beider Rufnummern zielführend. Wichtig ist, dass die Bearbeitung der Anrufe technisch und organisatorisch reibungslos integriert wird. Hierzu gehört neben dem digitalen Austausch von Patientendaten auch ein gemeinsamer Stand zu den verfügbaren Versorgungsstrukturen und deren Kapazitäten.

Darüber hinaus müssen unter beiden Rufnummern möglichst einheitliche Algorithmen und Standards eingesetzt werden. Eine zusätzliche Herausforderung ist, dass ein in den ILS eingesetztes Ersteinschätzungssystem zu den im INZ verwendeten Systemen kompatibel sein muss. Sowohl im Rettungsdienst als auch im ÄBD gibt es aktuell Bestrebungen, Systeme zur optimierten Patientensteuerung zu entwickeln (Lechleuthner et al. 2019; von Stillfried et al. 2019). Bei Vertretern der Notaufnahmen besteht eher die Tendenz, an etablierten

7 Der Begriff Integrierte Leitstelle bezeichnet im Bereich der Gefahrenabwehr üblicherweise die Zusammenführung von Dispositionseinrichtungen der Feuerwehr, des Katastrophenschutzes und des Rettungsdienstes. Diese Zusammenarbeit soll in den Vorschlägen des Sachverständigenrates beibehalten und um die weitergehende Integration des ärztlichen Bereitschaftsdienstes ergänzt werden.
Systemen wie dem Manchester Triage System festzuhalten (Kumle et al. 2019). Insgesamt zeigt sich dabei, dass die Vorstellungen zwischen der (prä-)klinischen Notfallmedizin und dem niedergelassenen Bereich stark divergieren (vgl. DGINA und DIVI 2019).

Keines der verfügbaren Systeme wird aktuell den Anforderungen der Patientensteuerung vollumfänglich gerecht (vgl. Möckel et al. 2019). So ist z. B. die Unsicherheit vor allem außerhalb der klar lebensbedrohlichen bzw. eindeutig nicht-dringlichen Fälle relativ groß (Hinson et al. 2019). Daher ist die zielgerichtete Weiterentwicklung und Evaluation der Systeme notwendig. Neuere, stärker IT-gestützte Ansätze könnten an dieser Stelle ansetzen und eine genauere Zuweisung ermöglichen. So konnten Algorithmen aus dem Bereich des maschinellen Lernens in verschiedenen (retrospektiven) Studien eine bessere Differenzierung der benötigten Versorgung (z. B. die anschließende Notwendigkeit intensivmedizinischer Behandlung) erreichen als ein klassisches Triagesystem wie der Emergency Severity Index. Die Systeme nutzen zur Klassifikation demographische Informationen, Leitsymptome (auch als Freitext), Vitalzeichen und Informationen aus der medizinischen Patientenakte (z. B. Levin et al. 2018; Raita et al. 2019). Auch ohne Informationen zur medizinischen Vorgeschichte, die zum Zeitpunkt der Ersteinschätzung ggf. nicht vorliegen, zeigten derartige Ansätze bessere Ergebnisse (Joseph et al. 2020). Sie sind daher vielversprechende Instrumente zur Unterstützung der Ersteinschätzung, müssen aber noch in praktischen Studien erprobt werden.

Welches System letztlich für den Einsatz geeignet ist, sollte auf Basis wissenschaftlicher Evaluierungen entschieden werden. Vorstellbar ist, dass unterschiedliche Ansätze mit klar definierten Schnittstellen in einem abgestuften System gemeinsam zur Anwendung kommen. So könnte ein möglichst einheitlich eingesetztes Ersteinschätzungssystem wie z. B. die inzwischen bundesweit von allen 116117-Leitstellen genutzte „Strukturierte medizinische 
Ersteinschätzung in Deutschland (SmED)“،8, ggf. mit Vor-Triage zur Feststellung der Dringlichkeit, genutzt und mit dem webbasierten ,Interdisziplinären Versorgungsnachweis (IVENA eHealth)“9, mit dem die Verfügbarkeit (stationärer und ambulanter) Kapazitäten in Kliniken bzw. Partnerpraxen in Echtzeit abgebildet wird, kombiniert werden.

Die eigentliche Filterung und Einschätzung der Anrufe sowie die sich anschließende Steuerung erfolgt durch erfahrene und speziell geschulte Rettungsdienstdisponenten. Um die bisher sehr heterogene Struktur der Leitstellen in Deutschland zu vereinheitlichen, sollte eine gezielte Personalentwicklung inkl. eines einheitlichen Berufsbildes vorangetrieben werden (vgl. Hackstein et al. 2015). Zusätzlich stehen in den ILS breit weitergebildete (Allgemein-) Ärzte mit Notfallerfahrung zur direkten telefonischen Abklärung komplexerer medizinischer Sachverhalte bereit.

Basierend auf der Ersteinschätzung der Fachkräfte in den ILS wird die jeweils individuell geeignete Versorgungsebene bzw. -struktur ausgewählt. Dies kann auch eine rein telefonische Beratung und Behandlung umfassen. Die wissenschaftliche Evidenz zur Auswirkung der telefonischen Ersteinschätzung und Beratung ist ambivalent (Boggan et al. 2020; van den Heede und van de Voorde 2016). Studien und Erfahrungen aus dem europäischen Ausland legen jedoch nahe, dass erfahrenes und geschultes (nicht zwingend ärztliches) Fachpersonal in vielen Fällen eine abschließende und sichere telefonische Versorgung sicherstellen kann (Boggan et al. 2020). Außerdem kann mit dem Ausbau der telefonischen Beratung Forderungen nach einem besseren Informationszugang Rechnung getragen werden (Köster-Steinebach 2019). Erfahrungen aus Pilotprojekten in Deutschland (z. B. ,,docdirekt“") und aus den ausgeweiteten Möglichkeiten zur telefonischen Behandlung während der Corona-Pandemie sollten in der Umsetzung berücksichtigt werden.

8 www.zi.de/smed/.

9 www.ivena.de.
Im Regelfall wird die ILS eine weitere nicht-telefonische Behandlung veranlassen. Hierfür bedarf es eines breiten Spektrums an Optionen. Für gehfähige Patientinnen und Patienten mit akutem Abklärungsbedarf werden kurzfristige Termine in den INZ (siehe - Abschn. 3.3.2) vergeben. Für wenig dringliche Akutfälle kann die ILS auf explizit ausgewiesene Partnerpraxen verweisen oder im Zusammenspiel mit den Terminservicestellen unmittelbar Termine bei niedergelassenen Ärztinnen und Ärzten vergeben. Eine begleitende (monetäre) Anreizsetzung zur Flexibilisierung und ggf. Ausweitung der Sprechstundenzeiten in Haus- und Facharztpraxen würde die Terminvergabe erleichtern und darüber hinaus zur Entlastung der Notfallversorgung beitragen (Morley et al. 2018). Außerdem sollte die Disposition von Hausbesuchen innerhalb festgelegter Fristen möglich sein. Entscheidet die ILS, dass ein Krankentransport anstelle einer Rettungsfahrt ausreichend ist, muss die Entscheidung einer ärztlichen Verordnung gleichstehen.

In manchen Fällen steht weniger ein notfallmedizinischer als vielmehr ein pflegerischer Aspekt im Vordergrund. Für diesen Fall wird die ILS neu einzurichtende Notpflegeteams alarmieren können. Diese können etwa in Pflegeheimen, aber auch bei Hausbesuchen eingesetzt werden, um bei bestimmten Einsätzen den Rettungsdienst und den ÄBD zu entlasten und pflegerische Aufgaben (z. B. einen Blasenkatheterwechsel) gezielt zu übernehmen. Auch die palliative Betreuung durch spezialisierte Palliativ-Care-Teams wird über die ILS koordiniert. So können Patientinnen und Patienten im finalen Krankheitsstadium auf Wunsch in ihrem heimischen Umfeld verbleiben, anstatt ungewollt in ein Krankenhaus transportiert zu werden.

Ein Beispiel für diesen weitgehenden Aufgabenausbau der Leitstellen findet sich in Österreich. Dort wurde eine telefonbasierte Erstberatungseinrichtung durch diplomierte Gesundheits- und Krankenpflegekräfte in der Landesleitstelle der Feuerwehr und des Rettungsdienstes im Land Vorarlberg (ca. 400.000 
Einwohner) angesiedelt. Ziel ist sowohl die Steuerung in geeignete Versorgungsstrukturen $^{10}$ als auch die direkte Beratung. Seit 2020 wird auch der ärztliche Bereitschaftsdienst durch die Leitstelle disponiert. Die Einbindung der verschiedenen Aufgaben erfolgt unter verschiedenen Rufnummern, die Bearbeitung geschieht jedoch in einer gemeinsamen Leitstelle. In der Gesundheitsberatung kann etwa einem Viertel der Anrufer rein telefonisch durch eine Beratung geholfen werden. Ansonsten werden unterschiedliche Dringlichkeitsstufen vom Notfall bis zur Routinebehandlung festgelegt oder konkrete nicht-ärztliche Versorgungspunkte benannt (Marxgut 2020).

Damit auch die ILS in Deutschland diese Aufgabenvielfalt erledigen können, sind gewisse Voraussetzungen zu schaffen. So ist ein flächendeckender Ausbau der digitalen Infrastruktur zwingend notwendig. Bisher verfügen die Leitstellen nicht durchgängig über aktuelle Informationen zu den Strukturen und Kapazitäten der Krankenhäuser bzw. Notaufnahmen. Um gezielt Versorgungspfade auszuwählen, müssen diese Informationen für alle an der Notfallversorgung Beteiligten erhoben werden und digital zugänglich sein. Auch eine einrichtungs- und sektorenübergreifende elektronische Patientenakte wäre für das Gelingen des Konzepts äußerst hilfreich, denn reibungslose Versorgung benötigt reibungslosen Informationsaustausch.

Mittelfristig ist außerdem eine stärkere horizontale Integration der Rettungsdienstbereiche und damit auch der Leitstellen anzustreben. Die bisherige oftmals kleinteilige Organisation bietet aus organisatorischer und ökonomischer Sicht Verbesserungsmöglichkeiten. Dabei geht es nicht darum, regionale Strukturen abzuschaffen und durch zentrale Lösungen zu ersetzen, sondern Organisationseinheiten so zu skalieren, dass die verfügbaren Ressourcen für eine bedarfsgerechte Versorgung optimal eingesetzt werden können.

10 Über das proprietäre „Emergency Communication Nurse System“. Ärztliches Personal steht für Rücksprachen bereit.
Schließlich ist eine wissenschaftliche Evaluation der ILS, die das konkrete Nutzungsverhalten der Patientinnen und Patienten, die sich ergebenden Steuerungseffekte und nach Möglichkeit auch die gesundheitlichen Outcomes untersucht, dringend anzuraten.

\subsubsection{Integrierte Notfallzentren}

Ausgehend von den Integrierten Leitstellen sind die neu zu schaffenden Integrierten Notfallzentren (INZ) die zentralen Anlaufstellen für die Versorgung von Notfällen. Sie integrieren die ambulanten und stationären Behandlungskapazitäten und bieten eine interdisziplinäre und sektorenübergreifende Versorgung „aus einer Hand“. Dazu werden ärztliche Bereitschaftspraxen und Notaufnahmen funktionell in eine gemeinsame Organisationseinheit am Standort einer Klinik integriert und deren Verfügbarkeit rund um die Uhr gewährleistet. Die INZ binden so die häufig isoliert agierenden Portalpraxen in ein gemeinsames Behandlungskonzept mit den Notaufnahmen ein. Die örtliche Zusammenführung in Verbindung mit einer rein medizinisch bedingten Versorgungssteuerung erscheint insbesondere sinnvoll, da ein hohes Substitutionspotenzial zwischen Leistungen des $\ddot{A B D}$ und der ambulanten Versorgung in Notaufnahmen besteht, das infolge der bisher fehlenden Steuerung auch praktisch genutzt wird (Krämer und Schreyögg 2019).

Um für alle Patientinnen und Patienten die Dringlichkeit sowie die geeignete Versorgungsstruktur zu bestimmen, ist der weiteren Versorgung eine zentrale Anlaufstelle (,ein gemeinsamer Tresen“) vorgeschaltet. Dort werden alle gehfähigen sowie die vom Rettungsdienst nicht als kritisch eingeschätzten Patientinnen und Patienten (ggf. erneut) auf Basis eines gemeinsamen bzw. zu den ILS voll kompatiblem Systems ersteingeschätzt (siehe - Abschn. 3.3.1). Ziel ist eine strukturierte und konsistente Entscheidung über das benötigte Versorgungssetting. 
Abhängig von Dringlichkeit und Bedarf kann dies die direkte Weiterleitung zur zentralen Notaufnahme im INZ mit einer ggf. stationären Weiterbehandlung oder die ambulante Behandlung durch den vor Ort integrierten ÄBD sein. Wird im Verlauf der Behandlung ein von der Ersteinschätzung abweichender Behandlungsbedarf festgestellt, können die Versorgungspfade ,,auf kurzem Dienstweg“ innerhalb des INZ angepasst werden. Fehlt die medizinische Dringlichkeit, kann die Ersteinschätzung auch einen Verweis auf die Untersuchung und Behandlung bei niedergelassenen Ärztinnen und Ärzten zur Folge haben. Im Zusammenhang mit einer direkten Terminvergabe ist die Behandlungssicherheit dennoch gewährleistet. Diese Möglichkeit erscheint notwendig, damit das INZ ausschließlich der Notfallversorgung dient und nicht als attraktives Zentrum einer schnellen und gut ausgestatteten Regelversorgung unnötige Inanspruchnahme induziert (Cooper et al. 2019).

Die Ersteinschätzung sollte im Idealfall durch breit qualifizierte Fachärzte (für Allgemeinmedizin) vorgenommen werden. Es gibt Hinweise dafür, dass die Einschätzung durch erfahrene Ärzte Wartezeiten und Wiedervorstellungen, stationäre Aufnahmeraten sowie Sterberaten verringern kann (Abdulwahid et al. 2016; Benabbas et al. 2020; Morley et al. 2018). Da die Einschätzung von ärztlichem und nicht-ärztlichem medizinischen Personal meist jedoch gut übereinstimmt (Pishbin et al. 2019), kann alternativ auch speziell geschultes Pflegepersonal diese Aufgabe übernehmen, sofern bei Bedarf ärztliche Expertise unmittelbar verfügbar ist. Wichtig ist, dass die Einschätzung weisungsunabhängig, rein auf Basis medizinischer Kriterien erfolgt.

Die interdisziplinäre und -professionelle Behandlung in den INZ setzt voraus, dass ärztliches Personal unterschiedlicher Facharztrichtungen und eine adäquate Ausstattung mit Pflegepersonal, wie sie auch für Notaufnahmen gefordert wird, verfügbar ist (Behringer et al. 2019). Neben unfallchirurgischen und internistischen Ärztinnen und Ärzte sind breit qualifizierte Generalisten wie Fachärzte für
Allgemeinmedizin - möglichst mit notfallmedizinischer Erfahrung - Basis der Personalausstattung. Eine solch breite Besetzung erlaubt es, unterschiedlichen patientenseitigen Anforderungen gerecht zu werden. Dringliche, aber ambulant behandelbare Patienten können mit geringem Diagnostikeinsatz versorgt werden, während die Behandlung komplexer und ggf. lebensbedrohlicher Fälle gewährleistet ist.

Die Rekrutierung von Personal, gerade in den Randzeiten, stellt viele Länder vor eine Herausforderung (Berchet und Nader 2016) und wird für die Schaffung dieser neuen Versorgungsstrukturen einen kritischen Erfolgsfaktor darstellen. Durch die Abschaffung von Doppelstrukturen und die Vermeidung nicht notwendiger organisatorischer Aufgaben kann jedoch bei ähnlichem Ressourceneinsatz eine höhere Qualität der Versorgung erzielt werden. Die hohe Attraktivität der neuen Arbeitsstrukturen kann ebenfalls zum Gelingen beitragen.

Analog zum Konzept in der stationären Notfallversorgung ist dazu eine gestufte Definition durch den G-BA mit personellen, infrastrukturellen und technischen Anforderungen $\mathrm{zu}$ beschließen. So können an einem Teil der INZ spezialisierte kinder- und jugendärztliche oder augenärztliche Behandlungskapazitäten vorgehalten werden und Zugriff auf einen psychiatrischen Kriseninterventionsdienst bestehen. Weitere Anforderungen sollten z.B. eine 24-stündige CT/MRT-Bereitschaft und Rückgriffmöglichkeiten auf spezialisierte Fachärzte der Kliniken (z. B. Radiologen oder Neurologen) umfassen. Höhere Stufen könnten die Verbindung mit im gleichen Haus bestehenden stationären Notfallkapazitäten, z. B. Chest Pain oder Stroke Units, voraussetzen. Zu den Anforderungen gehört auch, dass die stationäre Aufnahme von Patienten jederzeit sichergestellt ist; entweder durch eigene Bettenkapazitäten oder durch Kooperationsverträge mit anderen Krankenhäusern. Um unnötige stationäre Aufnahmen zu vermeiden und eine bestmögliche Abklärung im INZ zu erreichen, sind außerdem Kurzliegerstationen sinnvoll, in denen Patientinnen und Patienten ohne weitere Verlegung in die Klinik über 
Nacht überwacht werden können. So kann von der Basisnotfallversorgung bis hin zur Behandlung in spezialisierten Zentren mit besonderen Kapazitäten eine umfassende Versorgung auch in ländlichen Regionen gewährleistet werden.

Die Verantwortung für die INZ obliegt KV und Krankenhaus gemeinsam. Dabei sind verschiedene Ausgestaltungen mit unterschiedlicher Integrationstiefe - abhängig von den regionalen Strukturen - denkbar. Eine wünschenswerte Möglichkeit wäre die weitreichende organisatorische Integration, bei der die Leistungserbringung direkt durch das INZ mit eigenen Ressourcen und beim INZ angestellten Personal erfolgt. Ziel einer solchen Umsetzung ist, ein Vorbild für die sektorenübergreifende Versorgungssteuerung und Vergütung $\mathrm{zu}$ schaffen (siehe den Beitrag von Messerle und Schreyögg, $>$ Kap. 11 im gleichen Band). Denkbar sind jedoch auch Modelle mit geringerem Integrationsgrad. „Virtuelle INZ“ können die Leistungen des ÄBD und der zentralen Notaufnahme einkaufen und die benötigten Ressourcen vertraglich sicherstellen. Operationelles Kerngeschäft des INZ ist dann einzig der zentrale Tresen. Wesentliche Voraussetzung ist jedoch auch dann eine sektorenübergreifend einheitliche digitale Infrastruktur und der reibungslose Austausch digitaler Patientendaten. Nur unter dieser Bedingung kann die variable Inkorporation bereits bestehender Modelle und die Adaption an regionale Gegebenheiten flexibel gelingen.

Entsprechend dem Vorbildcharakter der neu strukturierten Notfallversorgung muss die bisher fragmentierte Planungszuständigkeit vereinheitlicht werden und die Kapazitätsund Standortplanung sektorenübergreifend aus einer Hand erfolgen. Die Gesamtplanung wird auf Landesebene verortet, aber unter Beachtung zentraler Vorgaben und mit Blick auf überregionale Koordination und Kooperation.

Die eigentliche Auswahl der Standorte und Vorgabe der benötigten Kapazitäten sollten gesetzlich weiterentwickelte Landesgremien nach § 90a SGB V (mit entsprechender personeller Unterstützung) vornehmen. Die Länder würden die Rolle der Rechtsaufsicht wahrneh- men und im Falle der Nichteinigung Ersatzvornahmen beschließen können. Die Kriterien für die Standort- und Stufenwahl müssen auf den vom G-BA beschlossenen Anforderungen basieren und u. a. Fallzahl, Strukturqualität und räumliche Abdeckung berücksichtigen. Es ist davon auszugehen, dass sowohl bei Maximalversorgern als auch bei ländlichen Krankenhäusern mit Sicherstellungszuschlag aufgrund der besonderen Strukturqualität bzw. der besonderen regionalen Bedeutung ein INZ errichtet würde. Für die Auswahl der Standorte ist aber ein transparentes und nachvollziehbares Verfahren durch den G-BA festzulegen, das durch die regionale Selbstverwaltung auf den lokalen Kontext angewendet wird. Eine denkbare Lösung ist auch, anstelle einer konkreten Standortplanung Kriterien für eine Ausschreibung zu definieren.

Krankenhäuser, an denen die abschließende Planung kein INZ vorsieht, nehmen nicht weiter an der Notfallversorgung teil. Dies erscheint auch infolge der unter Qualitäts- und Effizienzgesichtspunkten gebotenen Konzentration der Versorgung notwendig. Das de facto bereits heute konzentrierte Versorgungsgeschehen (zwei Drittel der Krankenhäuser erbringen $95 \%$ der stationären Notfallversorgung; G-BA 2018), die im internationalen Vergleich geringe durchschnittliche Auslastung der Notaufnahmen (von Stillfried et al. 2017) und die - rein rechnerisch - geringe Anzahl der zur Abdeckung des Versorgungsbedarfs benötigten Notfallzentren (Augurzky et al. 2019) sprechen für eine weitergehende Konzentration. Dies bedeutet im Gegenzug jedoch, dass den verantwortlichen Planungsgremien die Schließung von Notfallstandorten anvertraut werden muss. Da voraussichtlich vor allem Krankenhäuser in urbanen Verdichtungsräumen aus der Notfallversorgung ausscheiden würden, erscheint die Sorge vor fehlenden Kapazitäten, z. B. im Falle von Großschadensereignissen (vgl. Wurmb und Kowalzik 2019), unbegründet. Außerdem können ausscheidende Krankenhäuser durch Vereinbarungen, z. B. hinsichtlich der zielgerichteten Verlegung stationärer Patienten, in ein übergreifendes Ver- 
sorgungsnetz eingebunden werden. Entsprechende Wettbewerbsauswirkungen müssen dabei evaluiert werden.

Insgesamt ist zu erwarten, dass die Versorgungslandschaft der Notaufnahmen mittelfristig in die Strukturen der INZ migriert. Die hohen Qualitätsanforderungen und die finanzielle Aufwertung der Notfallversorgung (siehe unten) werden sicherstellen, dass ein höheres Qualitätsniveau als in der heutigen Situation erreicht werden kann. Die knappen Ressourcen, insbesondere im Bereich des qualifizierten Personals, werden konzentriert für eine hochwertige Notfallversorgung herangezogen.

Die Vergütung der INZ folgt als sektorenübergreifende Finanzierung den neu geschaffenen integrierten Strukturen. Leitgedanke ist, Transparenz über die Vergütung der Notfallversorgung herzustellen und Vergütungsanreize für eine optimale Patientenversorgung zu setzen. Das INZ wird hierfür als eigenständige wirtschaftliche Einheit etabliert. Die Vergütung setzt sich aus einer pauschalen Grundfinanzierung der Vorhaltekosten und einer Vergütung pro Fall zusammen. Die Höhe der Grundpauschale orientiert sich an der StufenEinordnung des INZ. Wie für den stationären Bereich zunehmend gefordert (Milstein und Schreyögg 2020), wird über eine solide Grundfinanzierung die Entkoppelung der Sicherstellung von den Fallzahlen erreicht und Mengenanreize oder Querfinanzierungen werden verhindert. Hierzu ist durch den G-BA eine angemessene Vorhaltepauschale in Abhängigkeit von der Versorgungstufe und ggf. geografischer Faktoren zu bestimmen. Die fallweise Vergütung erfolgt unabhängig von der Behandlungskomplexität. Lediglich die Beobachtung über Nacht wird durch einen Zuschlag separat finanziert. Ansonsten erhält das INZ unabhängig vom letztendlichen Ort der Behandlung - Bereitschaftsdienst oder Notaufnahme - eine Fallpauschale zur Deckung der Grenzkosten. Auch die Fallpauschalen sind vom G-BA festzulegen und könnten an das regionale Preisniveau angepasst werden.

Die eigentliche Kalkulation der Vergütung wird eher DRG- als EBM-ähnlich sein und sollte gemeinsam durch InBA und InEK erfolgen. Die Vorhaltepauschale wird auf die Krankenkassen nach (ggf. demographisch gewichtetem) Versichertenanteil aufgeteilt, die Fallpauschale direkt vom INZ mit der betroffenen Krankenkasse abgerechnet. Diese extrabudgetäre Vergütung wird durch eine Bereinigung der morbiditätsbedingten Gesamtvergütung sowie die sich aus der Versorgungsverlagerung ergebende Senkung der Klinikbudgets (z. B. durch den Wegfall pseudostationärer Fälle/Stundenfälle) refinanziert. Die Kritik an der Schaffung eines sogenannten ,dritten Sektors" mit eigenen Abrechnungsregeln und Strukturen (Korzilius 2019) geht insofern ins Leere, als dass nach diesem Verständnis wohl schon deutlich über zehn Sektoren existieren (vgl. die Liste der Regelungskreise in Leber und Wasem 2016). Doch eine gesonderte Vergütungsregel für gemeinsame Strukturen begründet keinen neuen Versorgungssektor. Jeglicher Fortschritt für eine am Patientenbedarf ausgerichtete sektorenübergreifende Versorgung und Vergütung (siehe auch $>$ Kap. 11, 12 und 13 in diesem Band) könnte andernfalls mit der interessengeleiteten Befürchtung eines „dritten Sektors“ negiert werden.

\subsubsection{Rettungsdienst}

Der Rettungsdienst stellt die dritte Säule der Notfallversorgung dar, wird jedoch bisher in der Sozialgesetzgebung als reine Transportleistung betrachtet. Hier sollte der Entwicklung der präklinischen Notfallmedizin Rechnung getragen und der Rettungseinsatz selbst als medizinische Leistung definiert werden. Dadurch würde der Fehlanreiz beseitigt, der durch die Verknüpfung der Fahrtkosten mit weiteren Leistungen besteht. Dies kann die anderen Bereiche der Notfallversorgung entlasten.

Durch die vorgestellten Reformen wird sich das Behandlungsspektrum des Rettungsdienstes stark verändern. Die Aufwertung der Leitstellen und der Zugriff auf zusätzliche Versorgungspfade wie die Hausbesuche des ÄBD, 
aber auch die individuelle telefonische Beratung führen dazu, dass sich die Versorgung im Rettungsdienst stärker auf lebensbedrohliche Situationen konzentrieren wird. Dies könnte neben der Entlastung auch zu einer wieder steigenden Attraktivität des Berufsbildes beitragen.

Dem Rettungsdienst wird außerdem zukünftig eine größere steuernde Rolle in der Versorgung zukommen. Stellt er vor Ort fest, dass entgegen der initialen Einschätzung der ILS kein Bedarf für die Versorgung im INZ oder im Krankenhaus vorliegt, kann der Rettungsdienst andere ihm angemessen erscheinende Ressourcen nachfordern. Als Option sollten auch der Transport des Patienten zu geeigneten ambulanten Partnerpraxen, die freiwillig verfügbare Notfallslots an die (elektronischen) Ressourcennachweissysteme melden, oder die ambulante Versorgung vor Ort in Frage kommen.

In diesem Zusammenhang sollten auch die Kompetenzen der Notfallsanitäter definiert und harmonisiert werden, damit sich die Befugnisse nicht von Kreis zu Kreis zum Teil erheblich unterscheiden und diesem neuen Berufsbild eine klare Versorgungsrolle zugewiesen wird. Auch die telemedizinische Zusammenarbeit mit fachärztlichem Personal sollte deutlich ausgebaut werden. So ist es möglich, Tätigkeiten - wie etwa die Medikamentengabe - unter Supervision an die Notfallsanitäter am Einsatzort zu delegieren und rechtlich abzusichern. Teilnehmer an einem Telenotarztmodell in Aachen stellten u. a. eine leitlinienkonformere Versorgung und die Erhöhung der Diagnosesicherheit fest (Schneiders et al. 2012). Weitere Studien legen nahe, dass der Telenotarzt die notärztlichen Kapazitäten entlasten und insbesondere für den ländlichen Raum eine vielversprechende Ergänzung in der präklinischen Notfallversorgung darstellen könnte (Süss et al. 2020).

Kernkompetenz des Rettungsdienstes ist die effektive und zeitkritische Versorgung in lebensbedrohlichen Situationen sowie der anschließende zeitgerechte Transport in eine geeignete Zielklinik (Bernhard et al. 2017). Hier kann es durch eine Konzentration der Versorgung in manchen Fällen zu längeren Zeitintervallen bis zur klinischen Versorgung kommen. Es existiert jedoch gute Evidenz dafür, dass auch bei längeren Transportwegen die Versorgung in besonders geeigneten Kliniken vorzuziehen ist (Ibanez et al. 2018). Dies kann - neben der für bestimmte Behandlungen benötigten speziellen Infrastruktur - darauf zurückzuführen sein, dass höhere Fallzahlen und die damit einhergehende Erfahrung eine besonders schnelle und sichere Durchführung erlauben (Nimptsch und Mansky 2017). Dementsprechend sehen notfallmedizinische Leitlinien den Transport in ein geeignetes Krankenhaus, nicht in das örtlich nächste, vor (Fischer et al. 2016). Die Feststellung der geeigneten Versorgungsstrategie und die darauf basierende Auswahl der Zielklinik bedarf einer hohen notärztlichen Kompetenz (Bernhard et al. 2017). Auch vor diesem Hintergrund ist eine stärkere telemedizinische Zusammenarbeit zu begrüßen.

Die Auswahl geeigneter Versorgungstrukturen ist nur möglich, wenn Informationen zu den Versorgungsmöglichkeiten und -kapazitäten stets aktuell zur Verfügung stehen. Hier ist analog zu den ILS ein flächendeckender Ausbau der digitalen Infrastruktur, z. B. durch Systeme wie IVENA, zu fördern. In dieser müssen nicht nur die Daten zu verfügbaren Krankenhäusern, sondern auch zu anderen Leistungserbringern abgebildet sein. Andernfalls ist weder die Anfahrt von Partnerpraxen noch die Nachforderung von Behandlungsressourcen umsetzbar. Außerdem ist der digitale und standardisierte Austausch von Einsatzund Patientendaten notwendig, um reibungslose Behandlungsübergänge zu gewährleisten. Die digitale Infrastruktur stellt also auch hier einen wichtigen Baustein der zukünftigen Notfallversorgung dar.

Schließlich sollte die Organisation und Finanzierung des Rettungsdienstes überarbeitet werden. Analog zur Krankenhausfinanzierung sind die Vorhaltekosten des Rettungsdienstes im Rahmen der Daseinsvorsorge des Staates aus Steuermitteln zu finanzieren. Nur die Betriebskosten sollten von den Krankenkas- 


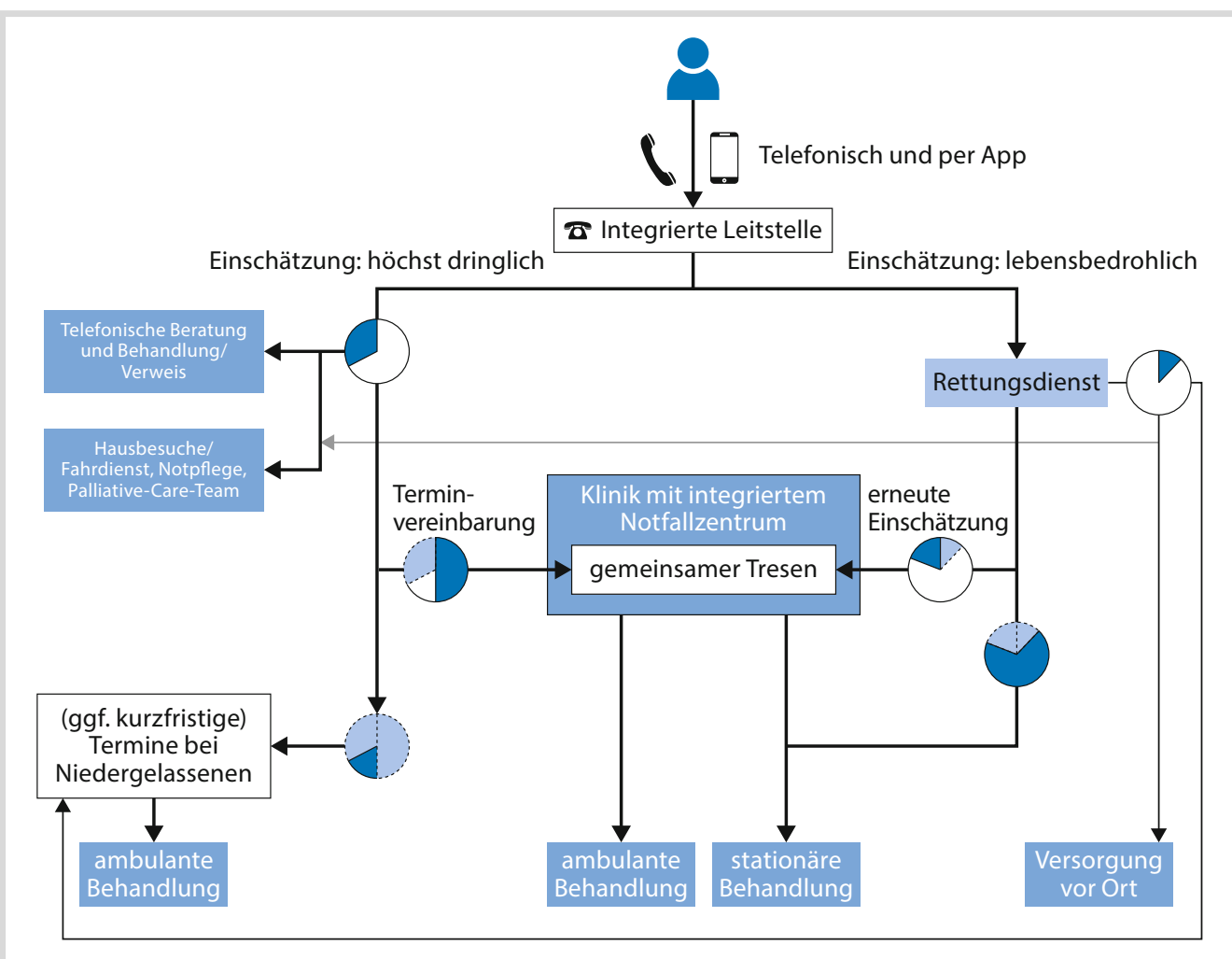

Krankenhaus-Report 2021

- Abb. 3.4 Patientenpfade in der zukünftigen Notfallversorgung

sen vergütet werden. Um den schleichenden Rückzug der Länder aus der finanziellen Verantwortung - ähnlich der Situation in der Krankenhausfinanzierung - zu verhindern, ist eine Rahmenregelung zur Finanzierung auf Bundesebene vorzuschreiben. Einzelheiten der Finanzierung sollten jedoch auf Landesebene geregelt werden, um weiterhin die Vereinbarung von Gesamtleistungsbudgets wie z. B. in Baden-Württemberg zu ermöglichen, welche die Kosten der Notfallversorgung insgesamt reduzieren können.

\subsubsection{Patientenpfade}

Die folgenden Einschätzungen stellen eine subjektive Zusammenstellung auf Basis ver- schiedener Quellen und Annahmen dar und sind angesichts der Komplexität der Veränderungen nur als grobe Orientierungswerte $\mathrm{zu}$ verstehen. Basierend auf dem vorgestellten Konzept könnte sich der Prozess der Notfallversorgung nach Kontaktierung der ILS ungefähr wie in $\bullet$ Abb. 3.4 darstellen.

Im Falle einer lebensbedrohlichen Situation rückt der (notarztbesetzte) Rettungsdienst aus. Trotz systematischer und strukturierter Ersteinschätzung wird ein kleiner Anteil der Patienten, voraussichtlich unter $10 \%{ }^{11}$, keinen Transport durch den Rettungsdienst benötigen, sondern kann abschließend vor Ort versorgt werden. Transportierte Patienten werden aufgrund der hohen Dringlichkeit in der Regel

11 Also unter dem heutigen Anteil in Baden-Württemberg, wo Daten dazu vorliegen (SQR-BW 2020). 
direkt in die stationäre Versorgung überstellt. In bestimmten Fällen kann aber auch eine ambulante Behandlung bzw. zunächst eine erneute Einschätzung im INZ oder der Transport in eine Partnerpraxis ausreichend sein. Aktuell wird etwa ein Drittel der mit dem Rettungsdienst kommenden Patienten in Notaufnahmen ambulant behandelt (basierend auf Zahlen für München; Trentzsch et al. 2019). Durch eine verbesserte Ersteinschätzung und Steuerung sowie die Änderung finanzieller Anreize im Rettungsdienst sollte dieser Anteil aber sinken.

Bei den höchstens als dringlich eingeschätzten Fällen zeigen Erfahrungen (Marxgut 2020) und Studien (Boggan et al. 2020), dass ein Viertel bis ein Drittel der Patienten telefonisch abschließend versorgt werden können; Verweise auf andere Versorgungsebenen (z. B. Apotheker) und die Disposition spezieller Hilfsangebote (z. B. Notfallpflege) ergänzen dieses Angebot. Für etwa ein Fünftel kann ein (ggf. kurzfristig) vereinbarter Termin in einer Praxis ausreichend sein (ebenfalls Marxgut 2020). Dementsprechend benötigt voraussichtlich höchstens die Hälfte der Fälle einen Termin für die akute ärztliche Versorgung in einem INZ, wo sie eine Versorgung aus einer Hand erhalten - unabhängig davon, ob letztlich ambulant oder stationär behandelt wird.

\subsection{Politische Entwicklungen}

Die Notfallversorgung ist seit langem Gegenstand gesundheitspolitischer Diskussionen. Dementsprechend werden wissenschaftliche Empfehlungen bereits seit vielen Jahren erarbeitet und vorgestellt (Slowik et al. 2018). Der Sachverständigenrat plädierte bereits 2003 und 2014 für die stärkere Vernetzung der Versorgungsbereiche und die Einrichtung Integrierter Leitstellen (SVR 2003, Ziff. 183 ff., 2014, Ziff. 552 ff.). 2018 veröffentlichte der Sachverständigenrat schließlich ein detailliertes Gesamtkonzept für die Zukunft der Notfallversorgung in Deutschland (SVR 2018, Ziff. 902 ff.), das in diesem Beitrag skizziert wurde.

Infolge der intensiven medialen Berichterstattung rückte die Notfallversorgung zunehmend auch in den Fokus der Öffentlichkeit und der Gesetzgeber wurde aktiv. So wurden die KVen mit dem GKV-Versorgungsstärkungsgesetz 2015 und dem Krankenhausstrukturgesetz 2016 schrittweise zu einer stärkeren Kooperation mit den Krankenhäusern angehalten. Das Krankenhausstrukturgesetz beauftragte darüber hinaus den G-BA, Regelungen zu einem gestuften System von stationären Notfallstrukturen zu erarbeiten.

Weitere Ideen wurden mit dem Terminservice- und Versorgungsgesetz (TSVG) umgesetzt. Zum einen wurden die 2016 eingeführten Terminservicestellen in ihrem Einsatzgebiet deutlich erweitert und mit der bundeseinheitlichen Bereitschaftsdienstnummer (116117) zusammengelegt. Diese sollen den Versicherten rund um die Uhr und auch digital in Akutfällen zur unmittelbaren ärztlichen Versorgung an eine Arztpraxis, eine Bereitschaftsdienstpraxis oder auch eine Notfallambulanz vermitteln. Zum anderen führte das TSVG finanzielle Anreize ein, die das Versorgungsangebot für Akutfälle im niedergelassenen Bereich erweitern sollen.

Nachdem im Dezember 2018 erste Eckpunkte zu einer umfassenden Reform der Notfallversorgung veröffentlicht wurden, folgte im Sommer 2019 ein Diskussionsentwurf. Nach zum Teil heftigen Diskussionen hat das Bundesministerium für Gesundheit erst im Januar 2020 - kurz vor Ausbruch der CoronaPandemie in Deutschland - den Referentenentwurf des Gesetzes zur Reform der Notfallversorgung vorgelegt (BMG 2020b). Dieser enthält die bereits im Koalitionsvertrag verabredeten Notfalleitstellen und integrierten Notfallzentren (CDU, CSU und SPD 2018, S. 100) sowie weitere Regelungen zum Rettungsdienst. Infolge der umfangreichen Diskussionen wurden die geplanten Reformen letztlich jedoch verschoben. Wie die Pläne in der nächsten Legislaturperiode aussehen werden, bleibt abzuwarten. 
- Gemeinsames Notfallleitsystem (GNL)

Die im Referentenentwurf als Gemeinsames Notfallleitsystem (GNL) bezeichnete Lösung soll zukünftig die zentrale Lotsenfunktion für die Notfallversorgung übernehmen. Wesentliches Merkmal der GNL ist ein gemeinsames Ersteinschätzungssystem für die 112 und die 116117. Die Disposition der Einsatzmittel erfolgt jedoch weiterhin getrennt: Während die 112 Rettungs- und Notarztwagen sowie zukünftig auch Krankentransporte disponieren kann, verbleiben für die 116117 die telefonische/telemedizinische Behandlung und Hausbesuche des ärztlichen Bereitschaftsdiensts. Über das GNL sollen die Leitstellen und alle an der Notfallversorgung Beteiligten umfassend digital vernetzt werden. Fokus ist neben dem Austausch einer digitalen Behandlungsdokumentation der Echtzeitzugriff auf die verfügbaren Ressourcen und Versorgungskapazitäten.

\section{- Integrierte Notfallzentren (INZ)}

Die INZ sollen, wie vom Sachverständigenrat empfohlen, an ausgewählten Standorten zentrale und rund um die Uhr verfügbare Anlaufstellen der Akut- und Notfallversorgung werden. In den INZ erhalten die Patientinnen und Patienten eine Ersteinschätzung und werden im Anschluss ambulant durch das INZ oder gezielt stationär in der Klinik versorgt. Die INZ werden unter Leitung der KV gemeinsam mit dem Krankenhaus betrieben. Hierfür sind Kooperationsvereinbarungen vorgesehen, welche die INZ räumlich so in das Krankenhaus einbinden, dass sie als erste Anlaufstelle im Notfall wahrgenommen werden.

Planungsvorgaben zur Anzahl und Standortauswahl der integrierten Notfallzentren, Richtlinien zur räumlichen, personellen und technisch-apparativen Ausstattung sowie Vorgaben zum Umfang der Notfallversorgung bestimmt der G-BA. Gestufte Vorgaben sind nicht vorgesehen. Die eigentliche Standortplanung der INZ erfolgt unter Beachtung der G-BA-Vorgaben durch erweiterte Landesausschüsse nach $§ 90$ SGB V. Bereits bestehende
Portalpraxen und die Notfallambulanzen der Krankenhäuser sollen sukzessive in INZ überführt werden.

Die Vergütung der INZ erfolgt extrabudgetär nach EBM. Hierzu sollen eine Grundpauschale sowie nach Schweregrad differenzierte Fallpauschalen aufgenommen werden.

\section{- - Rettungsdienst}

Der Rettungsdienst soll als eigener Leistungsbereich in das SGB V aufgenommen werden. Dabei werden sowohl die Versorgung vor Ort als auch die Rettungsfahrt als eigenständige Leistungen anerkannt. Es wird ferner festgehalten, dass durch den Rettungsdienst grundsätzlich die INZ anzufahren sind. Bei klar identifiziertem stationärem Behandlungsbedarf dürfen außerdem Krankenhäuser, die nach § 136c Absatz 4 SGB V an der stationären Notfallversorgung teilnehmen, direkt angesteuert werden. Weitergehende Festlegungen, etwa zur Finanzierung und zur Planung, sieht der Referentenentwurf nicht vor. Die ursprünglich im Diskussionsentwurf vorgesehenen bundesweiten Rahmenvorgaben sowie die Vorgaben zur Aufteilung der Finanzierung zwischen Bundesländern und Krankenkassen wurden gestrichen, wohl um eine Grundgesetzänderung zu vermeiden. Diese wäre gegen den Widerstand der Länder (Bensch 2019) nicht umsetzbar gewesen. Lediglich in der Gesetzesbegründung findet sich noch der Hinweis auf die verfassungsrechtlich mit der Länderkompetenz einhergehende Finanzierungsverantwortung.

Die Situation der Notfallsanitäter bleibt weiter offen. Nach unterschiedlichen Vorschlägen aus Landes- und Bundesebene (Deutscher Bundestag 2019a, 2019b) wird im MTAGesetz ein erneuter Versuch unternommen. Unter bestimmten Bedingungen sollen Notfallsanitäter eigenverantwortlich heilkundliche Maßnahmen invasiver Art durchführen können. Inwiefern dies abschließende Rechtssicherheit bringt und ob das Gesetz letztlich so beschlossen wird, ist zum Redaktionsschluss unklar. 


\subsection{Fazit}

Der Referentenentwurf zur Notfallversorgung wurde kurz vor einer der größten Krisen der Bundesrepublik veröffentlicht. Strukturelle Probleme des deutschen Gesundheitswesens wurden in der Krise besonders sichtbar und mussten quasi über Nacht zumindest provisorisch gelöst werden. Die fehlende Steuerung führte dazu, dass erst eine massive öffentliche Kommunikation die Patientenströme auf die 116117 aufmerksam machte. Verunsicherte Patienten trafen dennoch auf zum Teil unvorbereitete Krankenhäuser. Denn eine hohe Zahl an Krankenhausbetten bedeutet nicht automatisch, dass die Behandlung von COVID-19-Patienten gesichert wäre. Hierfür sind vielmehr spezielle Kenntnisse auf internistischen Stationen und die Verfügbarkeit von Intensivstationen mit Beatmungskapazitäten erforderlich. Dies ist vor allem bei Universitätskliniken und Maximalversorgern gegeben. Viele dieser Versorger haben zu wenige Intensivpflegekräfte, da sich das vorhandene Personal in Deutschland auf viele Kliniken verteilt, darunter auch auf Kliniken, die eine qualitativ hochwertige Notfallversorgung nicht erbringen können (Schreyögg 2020).

Im Verlauf der Krise führte die grassierende Angst und Unwissen über Versorgungsstrukturen mangels steuernder und anleitender Strukturen zur Vermeidung von Arztkontakten, möglicherweise mit gravierenden medizinischen Spätfolgen. Ansonsten zeigte sich insbesondere die fehlende digitale Vernetzung einmal mehr als großes Dilemma. So wurden unter anderem Zahlen gefaxt, per Telefon gemeldet oder händisch in Computer eingegeben. Eine bundesweite Kapazitätsmeldung wurde zwar schnell entwickelt, allerdings ergab sich in Bundesländern, in denen Krankenhäuser und Rettungsdienst bereits über IVENA vernetzt sind, ein doppelter Dokumentationsaufwand - von der Tatsache, dass die Ressourcen täglich manuell gemeldet werden mussten, ganz abgesehen. Auch die bereits bekannten Konflikte zur Vergütung der Notfallversor- gung traten erneut hervor (Deutsches Ärzteblatt 2020a; Ney 2020).

Vor diesem Hintergrund erscheint eine Reform der Notfallversorgung, wie sie oben dargestellt wurde und wie sie gesetzlich - trotz Verschiebung - für die Zukunft weiterhin geplant ist, einmal mehr notwendig. Eine bedarfsgerechte Steuerung der Patientenströme ist elementar für die Sicherstellung der Notfallversorgung. Diese kann nicht durch Kliniken oder niedergelassene Ärzte allein, sondern nur gemeinsam gewährleistet werden. Gut ausgestattete Krankenhäuser mit abgestuften Kapazitäten als zentrale Versorgungspunkte für schwerere Fälle müssen eng verknüpft mit einem ambulanten Versorgungsnetz zusammenarbeiten. Integrierte Leitstellen lenken die Versorgung - neben der telefonischen Beratung, die gerade während der Pandemie eine bedeutende Rolle einnahm - wo angemessen in die Strukturen der niedergelassenen Ärzte. In Integrierten Notfallzentren kann eine weitere Differenzierung der Versorgungsbedarfe vorgenommen werden. Durch den Aufbau klar abgestufter Strukturen mit adäquaten Ressourcen kann auch verhindert werden, dass Schwersterkrankte eine Vielzahl anderer Patienten in überfüllten Anlaufstellen infizieren.

Es sollte jedoch nicht vergessen werden, dass es weitere Bereiche gibt, die im $\mathrm{Zu}$ sammenhang mit einer Reform der Notfallversorgung Fortschritte bedürfen. $\mathrm{Zu}$ nennen sind hier insbesondere die sektorübergreifende Vergütung und die Digitalisierung im Gesundheitswesen (siehe Fraunhofer IAIS 2020 für Potenziale in der Notfallversorgung), aber auch eine Krankenhausstruktur- und -vergütungsreform ist notwendig. Mediale Ankündigungen, dass das Gesundheitssystem nach Corona zu reformieren sei (Deutsches Ärzteblatt 2020b), verheißen eine spannende Zeit.

\section{Literatur}

Abdulwahid MA, Booth A, Kuczawski M, Mason SM (2016) The impact of senior doctor assessment at tri- 
age on emergency department performance measures: systematic review and meta-analysis of comparative studies. Emerg Med J 33:504-513. https://doi.org/10. 1136/emermed-2014-204388

Augurzky B, Beivers A, Giebner M, Kirstein A (2015) Organisation der Notfallversorgung in Dänemark: Lösungsansätze für deutsche Probleme? In: Klauber J, Geraedts M, Friedrich J, Wasem J (Hrsg) Krankenhaus-Report 2015. Schwerpunkt: Strukturwandel. Schattauer, Stuttgart, S 77-97

Augurzky B, Beivers A, Breidenbach P, Budde R, Emde A, Haering A, Kaeding M, Roßbach-Wilk E, Straub N (2018) Notfallversorgung in Deutschland; Projektbericht im Auftrag der Kassenärztlichen Bundesvereinigung

Augurzky B, Beivers A, Breidenbach P, Haering A, Straub N (2019) Versorgungsplanung durch datenbasierte Marktraumanalysen am Beispiel von Notfallzentren. In: Klauber J, Geraedts M, Friedrich J, Wasem J (Hrsg) Krankenhaus-Report 2019. Das digitale Krankenhaus. Springer, Berlin Heidelberg, S 161-174

Baier N, Geissler A, Bech M, Bernstein D, Cowling TE, Jackson T, van Manen J, Rudkjøbing A, Quentin W (2019) Emergency and urgent care systems in Australia, Denmark, England, France, Germany and the Netherlands - Analyzing organization, payment and reforms. Health Policy 123:1-10. https://doi.org/10. 1016/j.healthpol.2018.11.001

BAND (Bundesvereinigung der Arbeitsgemeinschaften Notärzte Deutschlands) (2020) BAND-Stellungnahme zum „Entwurf eines Gesetzes zur Reform der Notfallversorgung“. Notarzt 36:75-77. https://doi.org/10. 1055/a-1107-6358

Behringer W, Buergi U, Christ M, Dodt C, Hogan B (2013) Fünf Thesen zur Weiterentwicklung der Notfallmedizin in Deutschland, Österreich und der Schweiz. Notfall Rettungsmed 16:625-626. https://doi.org/10. 1007/s10049-013-1821-8

Behringer W, Graeff I, Dietz-Wittstock M, Wrede CE, Mersmann J, Pin M, Kumle B, Möckel M, Gries A, Eisenburger P, Exadaktylos A, Dodt C (2019) Empfehlungen der notfallmedizinischen Gesellschaften DGINA, AAEM, SGNOR, DIVI, DGAI und DGIIN zur pflegerischen Besetzung von Klinischen Notfallzentren. Notfall Rettungsmed 22:330-333. https:// doi.org/10.1007/s10049-019-0585-1

Benabbas R, Shah R, Zonnoor B, Mehta N, Sinert R (2020) Impact of triage liaison provider on emergency department throughput: a systematic review and metaanalysis. Am J Emerg Med. https://doi.org/10.1016/j. ajem.2020.04.068

Bensch H (2019) Notfallversorgung - Widerstand gegen Spahns Notfallpläne wächst. https://www. bibliomedmanager.de/news/38921-widerstand-

gegen-spahns-notfallplaene-waechst. Zugegriffen: 25. Mai 2020
Berchet C, Nader C (2016) The organisation of out-ofhours primary care in OECD countries. OECD Health Working Papers Nr. 89

Bernhard M, Helm M, Lechleuthner A (2017) Erstversorgung vor Ort oder schnellstmöglicher Transportbeginn? Notfall Rettungsmed 20:579-585. https://doi. org/10.1007/s10049-017-0360-0

BMG (Bundesministerium für Gesundheit) (2020a) KG 2-Statistik. Leistungsfälle bei Rettungsfahrten und Krankentransporten der Versicherten der gesetzlichen Krankenversicherung. In: www.gbe-bund.de (Gesundheitsversorgung > Beschäftigte und Einrichtungen der Gesundheitsversorgung $>$ Rettungsdienste, Krankentransportwesen)

Bundesministerium für Gesundheit (2020b) Referentenentwurf zur Reform der Notfallversorgung

Boggan JC, Shoup JP, Whited JD, van Voorhees E, Gordon AM, Rushton S, Lewinski AA, Tabriz AA, Adam S, Fulton J, Kosinski AS, van Noord MG, Williams JW, Goldstein KM, Gierisch JM (2020) Effectiveness of acute care remote triage systems: a systematic review. J Gen Intern Med. https://doi.org/10.1007/ s11606-019-05585-4

Breckner A, Roth C, Wensing M, Paulus J (2020) Quo vadis 116117? Bundesweiter Überblick über den Status quo und aktuelle Veränderungen. Gesundheitswesen 82:324-327. https://doi.org/10.1055/a-1075-2330

Breuer F, Pommerenke C, Lamers A, Schloack S, Langhammer S, Dahmen J, Jüttner JP, Plock G, Drescher S, Poloczek S (2020) Generaldelegation von heilkundlichen Maßnahmen an Notfallsanitäter - Umsetzung im Land Berlin. Notfall Rettungsmed. https://doi.org/10. 1007/s10049-020-00683-x

CDU, CSU, SPD (2018) Ein neuer Aufbruch für Europa. Eine neue Dynamik für Deutschland. Ein neuer Zusammenhalt für unser Land; Koalitionsvertrag zwischen CDU, CSU und SPD. 19. Legislaturperiode

Cooper A, Davies F, Edwards M, Anderson P, Carson-Stevens A, Cooke MW, Donaldson L, Dale J, Evans BA, Hibbert PD, Hughes TC, Porter A, Rainer T, Siriwardena A, Snooks H, Edwards A (2019) The impact of general practitioners working in or alongside emergency departments: a rapid realist review. Bmj Open 9:e24501. https://doi.org/10.1136/ bmjopen-2018-024501

Destatis (Statistisches Bundesamt) (2017) Grunddaten der Krankenhäuser. Fachserie 12 Reihe 6.1.1

Destatis (Statistisches Bundesamt) (2019) Vollstationäre Patientinnen und Patienten in Krankenhäusern (DRGStatistik, Eckdaten). In: www.gbe-bund.de (Datenquellen des Statistischen Bundesamtes > Datenquelle: DRG-Statistik PEPP-Statistik)

Deutscher Bundestag (2019a) Ausschuss für Gesundheit; Ausschussdrucksache 19(14)108.1. Änderungsantrag 1 der Fraktionen der CDU/CSU und SPD zu BT-Drs. $19 / 13825$ 
Deutscher Bundestag (2019b) Gesetzentwurf des Bundesrates Entwurf eines Gesetzes zur Änderung des Notfallsanitätergesetzes. Drucksache 19/15274

Deutscher Bundestag (2020) Das Berufsbild der Notfallsanitäterin und des Notfallsanitäters unter besonderer Berücksichtigung der Ausbildungszielbestimmung des $\S 4$ Abs. 2 Nr. 2 Buchstabe c Notfallsanitätergesetz; Bundesrechtliche Vorgaben und Umsetzung in den Bundesländern. Aktualisierung der Arbeit WD 9-042/16

Deutsches Ärzteblatt (Hrsg) (2020a) Neuer Streit um Notfallversorgung. https://www. aerzteblatt.de/nachrichten/114950/Neuer-Streit-umNotfallversorgung. Zugegriffen: 24. Juli 2020

Deutsches Ärzteblatt (Hrsg) (2020b) Söder: Deutsches Gesundheitssystem muss nach Corona reformiert werden. https://www.aerzteblatt.de/nachrichten/111878/ Soeder-Deutsches-Gesundheitssystem-muss-nachCorona-reformiert-werden. Zugegriffen: 3. Sept. 2020

DGINA (Deutsche Gesellschaft Interdisziplinäre Notfallund Akutmedizin), DIVI (Deutsche Interdisziplinäre Vereinigung für Intensiv- und Notfallmedizin) (2019) Gemeinsame Stellungnahme DGINA und DIVI zur derzeitigen Entwicklung. https://www.dgina.de/news/ gemeinsame-stellungnahme-dgina-und-divi-zurderzeitigen-entwicklung-ersteinschatzung_82

DKG (Deutsche Krankenhausgesellschaft e.V.) (2019) Bestandsaufnahme zur Krankenhausplanung und Investitionsfinanzierung in den Bundesländern (Stand: Dezember 2019)

Dräther H, Schäfer T (2017) Die ambulante Notfallversorgung in Notfallambulanzen und bei Vertragsärzten im Zeitraum 2009 bis 2014. In: Klauber J, Geraedts M, Friedrich J, Wasem J (Hrsg) Krankenhaus-Report 2017. Zukunft gestalten. Schattauer, Stuttgart, S 25-40

Europäische Kommission (2020) Implementation of the single European emergency number 112 - Results of the thirteenth data-gathering round. Communications Committee. Working Document COCOM20-05

Fischer M, Kehrberger E, Marung H, Moecke H, Prückner S, Trentzsch H, Urban B (2016) Eckpunktepapier 2016 zur notfallmedizinischen Versorgung der Bevölkerung in der Prähospitalphase und in der Klinik. Notfall Rettungsmed 19:387-395. https://doi.org/10. 1007/s10049-016-0187-0

Fløjstrup M, Bogh SB, Henriksen DP, Bech M, Johnsen SP, Brabrand M (2020) Increasing emergency hospital activity in Denmark, 2005-2016: a nationwide descriptive study. Bmj Open 10:e31409. https://doi. org/10.1136/bmjopen-2019-031409

Foster H, Moffat KR, Burns N, Gannon M, Macdonald S, O'Donnell CA (2020) What do we know about demand, use and outcomes in primary care out-of-hours services? A systematic scoping review of internatio- nal literature. Bmj Open 10:e33481. https://doi.org/ 10.1136/bmjopen-2019-033481

Fraunhofer IAIS (Fraunhofer-Institut für Intelligente Analyse- und Informationssysteme) (2020) Whitepaper »Künstliche Intelligenz im Krankenhaus«

Gemeinsamer Bundesausschuss (2018) Neue G-BA-Regelung zur stationären Notfallversorgung: Sichere Erreichbarkeit, verbesserte Qualität und zielgenaue Finanzierung; Pressemitteilung. https://www.g-ba.de/ presse/pressemitteilungen/744/. Zugegriffen: 7. Mai 2020

Gonçalves-Bradley D, Khangura JK, Flodgren G, Perera R, Rowe BH, Shepperd S (2018) Primary care professionals providing non-urgent care in hospital emergency departments. Cochrane Database Syst Rev. https://doi.org/10.1002/14651858.CD002097.pub4

Graff L (1999) Overcrowding in the ED: An international symptom of health care system failure. Am J Emerg Med 17:208-209. https://doi.org/10.1016/ S0735-6757(99)90064-5

Günther A, Schmid S, Bruns A, Kleinschmidt T, Bartkiewicz T, Harding U (2017) Ambulante Kontakte mit dem Rettungsdienst. Notfall Rettungsmed 20:477-485. https://doi.org/10.1007/s10049-0170268-8

Haas C, Larbig M, Schöpke T (2015) Gutachten zur ambulanten Notfallversorgung im Krankenhaus; Fallkostenkalkulation und Strukturanalyse

Hackstein A, Lenz W, Marung H (2015) Personalqualifikation in der Leitstelle. Notfall Rettungsmed 18:553-559. https://doi.org/10.1007/s10049-0150048-2

Hinson JS, Martinez DA, Cabral S, George K, Whalen M, Hansoti B, Levin S (2019) Triage performance in emergency medicine: a systematic review. Ann Emerg Med 74:140-152. https://doi.org/10.1016/ j.annemergmed.2018.09.022

Ibanez B, James S, Agewall S, Antunes MJ, BucciarelliDucci C, Bueno H, Caforio ALP, Crea F, Goudevenos JA, Halvorsen S, Hindricks G, Kastrati A, Lenzen MJ, Prescott E, Roffi M, Valgimigli M, Varenhorst C, Vranckx P, Widimský P (2018) 2017 ESC Guidelines for the management of acute myocardial infarction in patients presenting with ST-segment elevation: The Task Force for the management of acute myocardial infarction in patients presenting with ST-segment elevation of the European Society of Cardiology (ESC). Eur Heart J 39:119-177. https://doi.org/10. 1093/eurheartj/ehx393

Joseph JW, Leventhal EL, Grossestreuer AV, Wong ML, Joseph LJ, Nathanson LA, Donnino MW, Elhadad N, Sanchez LD (2020) Deep-learning approaches to identify critically Ill patients at emergency department triage using limited information. J Am Coll Emerg Physicians Open. https://doi.org/10.1002/ emp2.12218 
Karagiannidis C, Kluge S, Riessen R, Krakau M, Bein T, Janssens U (2019) Auswirkungen des Pflegepersonalmangels auf die intensivmedizinische Versorgungskapazität in Deutschland. Med Klin Intensivmed Notfmed 114:327-333. https://doi.org/10.1007/ s00063-018-0457-3

Kassenärztliche Bundesvereinigung (2020) KBV Versichertenbefragung 2019; Anlaufstellen Notfallversorgung. https://gesundheitsdaten.kbv.de/cms/ html/36643.php. Zugegriffen: 12. Sept. 2020

kkvd (Katholischer Krankenhausverband Deutschlands) (2017) Notfallversorgung in Deutschland; Forsa-Umfrage. https://kkvd.de/wp-content/uploads/ downloads/Daten_Forsa.pdf. Zugegriffen: 12. Sept. 2020

Korzilius H (2019) Notfallreform: Gestritten wird über das „Wie“, nicht über das „Ob“. Dtsch Arztebl 116:A-1579

Köster-Steinebach I (2019) Anforderungen aus Patientensicht. Kooperationstagung Rettungsleitstellen, Berlin, 16. Jan. 2019

Krämer J, Schreyögg J (2019) Substituting emergency services: primary care vs. hospital care. Health Policy 123:1053-1060. https://doi.org/10. 1016/j.healthpol.2019.08.013

Krämer J, Schreyögg J, Busse R (2019) Classification of hospital admissions into emergency and elective care: a machine learning approach. Health Care Manag Sci 22:85-105. https://doi.org/10.1007/s10729017-9423-5

Kumle B, Hirschfeld-Warneken A, Darnhofer I, Busch HJ (2019) Telefon-Triage und klinische Ersteinschätzung in der Notfallmedizin zur Patientensteuerung. Notfall Rettungsmed 22:568-577. https://doi.org/10. 1007/s10049-019-0622-0

Leber W-D, Wasem J (2016) Ambulante Krankenhausleistungen - ein Überblick, eine Trendanalyse und einige ordnungspolitische Anmerkungen. In: Klauber J, Geraedts M, Friedrich J, Wasem J (Hrsg) Krankenhaus-Report 2016. Schwerpunkt: Ambulant im Krankenhaus. Schattauer, Stuttgart, S 3-28

Lechleuthner A (2017) Gestuftes Versorgungssystem im Rettungsdienst (GVS) (Working Paper)

Lechleuthner A, Wesolowski M, Brandt S (2019) Gestuftes Versorgungssystem im Kölner Rettungsdienst. Notfall Rettungsmed 22:598-607. https://doi.org/10. 1007/s10049-019-00644-z

Levin S, Toerper M, Hamrock E, Hinson JS, Barnes S, Gardner H, Dugas A, Linton B, Kirsch T, Kelen G (2018) Machine-learning-based electronic triage more accurately differentiates patients with respect to clinical outcomes compared with the emergency severity index. Ann Emerg Med 71:565-574.e2. https://doi. org/10.1016/j.annemergmed.2017.08.005

Luiz T, Dittrich S, Pollach G, Madler C (2017) Kenntnisstand der Bevölkerung über Leitsymptome kardiovaskulärer Notfälle und Zuständigkeit und Erreichbar- keit von Notrufeinrichtungen: Ergebnisse der KZENStudie in der Westpfalz. Anaesthesist 66:840-849. https://doi.org/10.1007/s00101-017-0367-4

Luiz T, Marung H, Pollach G, Hackstein A (2019) Implementierungsgrad der strukturierten Notrufabfrage in deutschen Leitstellen und Auswirkungen ihrer Einführung. Anaesthesist 68:282-293. https://doi.org/10. 1007/s00101-019-0570-6

Mann V, Mann STW, Müller M, Edeler B, Sander M, Brenck F (2020) Standardisierte Handlungsanweisungen für (invasive) heilkundliche Maßnahmen durch Notfallsanitäter. Notfall Rettungsmed 23:16-22. https://doi.org/10.1007/s10049-018-0556-y

Marxgut S (2020) Die Leitstelle als Wegweiser im Gesundheitssystem. Notfall Rettungsmed. https://doi. org/10.1007/s10049-020-00777-6

Miles J, O'Keeffe C, Jacques R, Stone T, Mason S (2017) 17 Exploring ambulance conveyances to the emergency department: a descriptive analysis of non-urgent transports. Emerg Med J 34:A872-A873. https://doi. org/10.1136/emermed-2017-207308.17

Milstein R, Schreyögg J (2020) Bedarfsgerechte Gestaltung der Krankenhausvergütung - Reformvorschläge unter der Berücksichtigung von Ansätzen anderer Staaten. Techniker Krankenkasse (TK), Hamburg

Ministeriet for Sundhed og Forebyggelse (2014) Faglig Gennemgang Af Akutmodtagelserne

Möckel M, Reiter S, Lindner T, Slagman A (2019) „,Triagierung" - Ersteinschätzung von Patienten in der zentralen Notaufnahme: Eine Übersicht mit systematischem Review. Med Klin Intensivmed Notfmed. https://doi.org/10.1007/s00063-019-0589-0

Moellekaer A, Duvald I, Obel B, Madsen B, Eskildsen J, Kirkegaard H (2019) The organization of Danish emergency departments. Eur J Emerg Med 26:295-300. https://doi.org/10.1097/ MEJ.0000000000000554

Morley C, Unwin M, Peterson GM, Stankovich J, Kinsman L (2018) Emergency department crowding: a systematic review of causes, consequences and solutions. PLoS ONE 13:e203316. https://doi.org/10. 1371/journal.pone.0203316

Ney R (2020) DKG kritisiert fehlende Kostensicherheit für COVID-19-Tests in Kliniken. Ärzte Zeitung. https://www.aerztezeitung.de/Politik/DGK-kritisiertfehlende-Kostensicherheit-fuer-COVID-19-Tests-inKliniken-408547.html. Zugegriffen: 14. Aug. 2020

Niedersächsischer Landtag (2015) Antworten auf Mündliche Anfragen gemäß $\$ 47$ der Geschäftsordnung des Niedersächsischen Landtages - Drs. 17/4530. Drucksache $17 / 4595$

Nimptsch U, Mansky T (2017) Hospital volume and mortality for 25 types of inpatient treatment in German hospitals: observational study using complete national data from 2009 to 2014. Bmj Open 7:e16184. https:// doi.org/10.1136/bmjopen-2017-016184 
Oslislo S, Heintze C, Schmiedhofer M, Möckel M, Schenk L, Holzinger F (2019) How to decide adequately? Qualitative study of GPs' view on decision-making in self-referred and physician-referred emergency department consultations in Berlin, Germany. Bmj Open 9:e26786. https://doi.org/10.1136/ bmjopen-2018-026786

Pishbin E, Ebrahimi M, Mirhaghi A (2019) Do physicians and nurses agree on triage levels in the emergency department? A meta-analysis. Notfall Rettungsmed 22:379-385. https://doi.org/10.1007/ s10049-019-0580-6

PwC (PricewaterhouseCoopers) (2019) Notaufnahmen in Not? Eine Studie zur Notfallversorgung in Deutschland 2019

Raita Y, Goto T, Faridi MK, Brown DFM, Camargo CA, Hasegawa K (2019) Emergency department triage prediction of clinical outcomes using machine learning models. Crit Care 23:64. https://doi.org/10.1186/ s13054-019-2351-7

Reinhold AK, Greiner F, Schirrmeister W, Walcher F, Erdmann B (2020) Der Notfall ,geht“ ins Krankenhaus: Eine Befragung von Patienten mit niedriger Dringlichkeit in einer Notfallaufnahme mit regionaler Alleinstellung. Med Klin Intensivmed Notfmed. https://doi.org/10.1007/s00063-020-00681-4

Roßbach-Wilk E, Beivers A, Dodt C (2019) Patientensteuerung von Notfallpatienten mit niedrigem Gesundheitsrisiko. Notfall Rettungsmed 22:561-567. https://doi.org/10.1007/s10049-019-0618-9

Scherer M, Lühmann D, Kazek A, Hansen H, Schäfer I (2017) Patients attending emergency departments. Dtsch Arztebl Int 114:645-652. https://doi.org/10. 3238/arztebl.2017.0645

Schmidt FM, Wildner M (2019) Übersicht über die Organisation des vertragsärztlichen Bereitschaftsdienstes in Deutschland: Auf welche Basis bauen Krankenhausstrukturgesetz und Versorgungsstärkungsgesetz auf? Gesundheitswesen 81:e133-e140. https:// doi.org/10.1055/a-0725-8193

Schmiedel R, Behrendt H (2019) Analyse des Leistungsniveaus im Rettungsdienst für die Jahre 2016 und 2017. Berichte der Bundesanstalt für Straßenwesen

Schmiedhofer M, Searle J, Slagman A, Frick J, Ruhla S, Möckel M (2017) Bedeutung der Notaufnahme für die ambulante medizinische Versorgung in einer ruralen Region in Sachsen-Anhalt: Qualitative Befragung von Patienten und Hausärzten. Dtsch Med Wochenschr 142:e61-e73. https://doi.org/10.1055/s-0043-100639

Schneiders M-T, Herbst S, Schilberg D, Isenhardt I, Jeschke S, Fischermann H, Bergrath S, Rossaint R, Skorning M (2012) Telenotarzt auf dem Prüfstand. Notfall Rettungsmed 15:410-415. https://doi.org/10. 1007/s10049-011-1535-8

Schreyögg J (2020) Corona-Krise trifft auf Strukturprobleme im Gesundheitswesen. Wirtschaftsdienst 100:226-227
Searle J, Muller R, Slagman A, Schäfer C, Lindner T, Somasundaram R, Frei U, Möckel M (2015) Überfüllung der Notaufnahmen. Notfall Rettungsmed 18:306-315. https://doi.org/10.1007/s10049-015-0011-2

Slowik M, Wehner C, Dräther H, Fahlenbrach C, Richard S (2018) Sektorübergreifende Neuordnung der Notfallversorgung. In: Klauber J, Geraedts M, Friedrich J, Wasem J (Hrsg) Krankenhaus-Report 2018. Schwerpunkt: Bedarf und Bedarfsgerechtigkeit. Schattauer, Stuttgart, S 233-257

Somasundaram R, Geissler A, Leidel BA, Wrede CE (2018) Beweggründe für die Inanspruchnahme von Notaufnahmen - Ergebnisse einer Patientenbefragung. Gesundheitswesen 80:621-627. https://doi.org/ 10.1055/s-0042-112459

Søvsø MB, Huibers L, Bech BH, Christensen HC, Christensen MB, Christensen EF (2020) Acute care pathways for patients calling the out-of-hours services. BMC Health Serv Res 20:146. https://doi.org/10. 1186/s12913-020-4994-0

SQR-BW (Stelle zur trägerübergreifenden Qualitätssicherung im Rettungsdienst Baden-Württemberg) (2020) Qualitätsbericht; Berichtsjahr 2019. Rettungsdienst Baden-Württemberg

von Stillfried D, Czihal T, Erhart M (2017) Rolle der Krankenhäuser in der Notfallversorgung in Deutschland: Daten belegen massiven Reformbedarf. ZiPaper 11/2017

von Stillfried D, Czihal T, Meer A (2019) Sachstandsbericht: Strukturierte medizinische Ersteinschätzung in Deutschland (SmED). Notfall Rettungsmed 22:578-588. https://doi.org/10.1007/s10049-0190627-8

Süss R, Dewenter C, Ekinci A, Laslo T, Fleßa S (2020) Das Telenotarztsystem - Potentiale für die präklinische Notfallversorgung im ländlichen Raum. Gesundh Ökon Qual Manag. https://doi.org/10.1055/a-11002639

SVR (Sachverständigenrat für die Konzertierte Aktion im Gesundheitswesen) (2003) Finanzierung, Nutzerorientierung und Qualität. Nomos, Baden-Baden

Sachverständigenrat zur Begutachtung der Entwicklung im Gesundheitswesen (2014) Bedarfsgerechte Versorgung; Perspektiven für ländliche Regionen und ausgewählte Leistungsbereiche. Huber, Bern

SVR (Sachverständigenrat zur Begutachtung der Entwicklung im Gesundheitswesen) (2018) Bedarfsgerechte Steuerung der Gesundheitsversorgung; Gutachten 2018. Medizinisch Wissenschaftliche Verlagsgesellschaft, Berlin

The Capital Region of Denmark (2019) More patients in the Capital Region of Denmark to be treated at home and avoid acute hospital admissions. https://www.regionh.dk/english/press-and-news/ latest-news/Pages/More-patients-in-the-CapitalRegion-of-Denmark-to-be-treated-at-home-and- 
avoid-acute-hospital-admissions.aspx. Zugegriffen: 8. Juni 2020

Trentzsch H, Dodt C, Gehring C, Veser A, Jauch K-W, Prückner S (2019) Analyse der Behandlungszahlen in den Münchener Notaufnahmen des Jahres 2013/2014. Gesundheitswesen. https://doi.org/10. 1055/a-0925-8989

van den Heede K, van de Voorde C (2016) Interventions to reduce emergency department utilisation: a review of reviews. Health Policy 120:1337-1349. https://doi. org/10.1016/j.healthpol.2016.10.002

VIVE (Det Nationale Forsknings- og Analysecenter for Velfærd) (2018) Regionale lægevagter og Akuttelefonen 1813

Walter C, Fischer F (2017) Interdisziplinärer Versorgungsnachweis (IVENA): Verbesserung der Notfallversorgung durch E-Health? Notarzt 33:50-51. https://doi. org/10.1055/s-0043-105634
Wolcott BW (1979) What is an emergency? Depends on whom you ask. J Am Coll Emerg Physicians 8:241-243. https://doi.org/10.1016/S03611124(79)80188-4

Wurmb T, Kowalzik B (2019) Fokus Krankenhäuser: Neustrukturierung der Notfallversorgung in Deutschland - Großschadensereignisse auf dem Schirm? In: Visarius J, Kloepfer A (Hrsg) iXForum. Gesundheitspolitik in der Diskussion Institut für GesundheitssystemEntwicklung, S 10-16

$\mathrm{Zi}$ (Zentralinstitut für die kassenärztliche Versorgung) (2019a) Grafik des Monats; Juli 2019. https://www. zi.de/presse/grafikdesmonats/. Zugegriffen: 24. Juli 2020

$\mathrm{Zi}$ (Zentralinstitut für die kassenärztliche Versorgung) (2019b) Zahlen zur ambulanten Notfallversorgung in Deutschland. https://www.zi.de/fileadmin/images/ content/PDFs_alle/Broschuere_final.pdf. Zugegriffen: 24. Aug. 2020

Open Access Dieses Kapitel wird unter der Creative Commons Namensnennung 4.0 International Lizenz (http:// creativecommons.org/licenses/by/4.0/deed.de) veröffentlicht, welche die Nutzung, Vervielfältigung, Bearbeitung, Verbreitung und Wiedergabe in jeglichem Medium und Format erlaubt, sofern Sie den/die ursprünglichen Autor(en) und die Quelle ordnungsgemäß nennen, einen Link zur Creative Commons Lizenz beifügen und angeben, ob Änderungen vorgenommen wurden.

Die in diesem Kapitel enthaltenen Bilder und sonstiges Drittmaterial unterliegen ebenfalls der genannten Creative Commons Lizenz, sofern sich aus der Abbildungslegende nichts anderes ergibt. Sofern das betreffende Material nicht unter der genannten Creative Commons Lizenz steht und die betreffende Handlung nicht nach gesetzlichen Vorschriften erlaubt ist, ist für die oben aufgeführten Weiterverwendungen des Materials die Einwilligung des jeweiligen Rechteinhabers einzuholen.

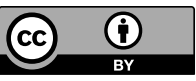

